\title{
Resonance Raman Excitation Profiles of Fe(II)-Terpyridine Complexes: Electronic Effects of Ligand Modifications
}

Jan Kožíšek ${ }^{1}$, Jan Svoboda ${ }^{2}$, Jiři Zedník ${ }^{1}$, Blanka Vlčková ${ }^{1}$, Ivana Šloufová ${ }^{1}$

${ }^{1}$ Charles University, Faculty of Science, Department of Physical and Macromolecular Chemistry, Hlavova 2030, 12840 Prague 2, Czech Republic

${ }^{2}$ Institute of Macromolecular Chemistry, Czech Academy of Sciences, Heyrovskeho nam. 2, 16206 Prague 6, Czech Republic

*E-mail for Ivana Šloufová: sloufovi@natur.cuni.cz

Supporting information: 

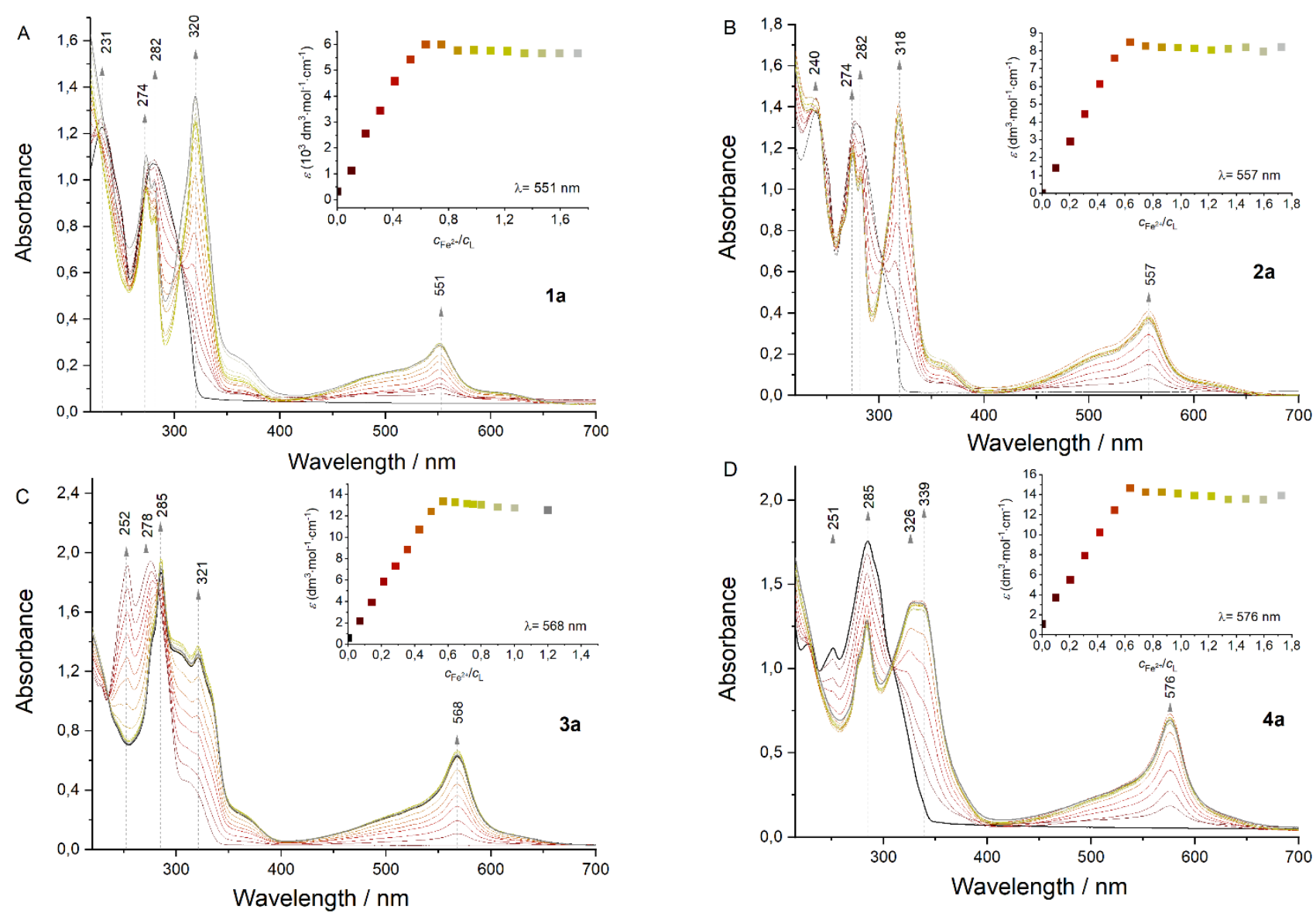

Figure S1 - Changes in UV/vis spectra accompanied titrations of ligands $\mathbf{1}-\mathbf{4}$ with Fe(II) ions. Initial ligand concentration was $5 \cdot 10^{-5} \mathrm{M}$ (methanol for 1, 2, 4 and mixture acetonitrile-methanol 1:1 for 3). Titration was performed with $100 \mu$ l steps of $5 \cdot 10^{-4} \mathrm{M}$ solution of $\mathrm{FeSO}_{4}$ in in methanol, room temperature. 


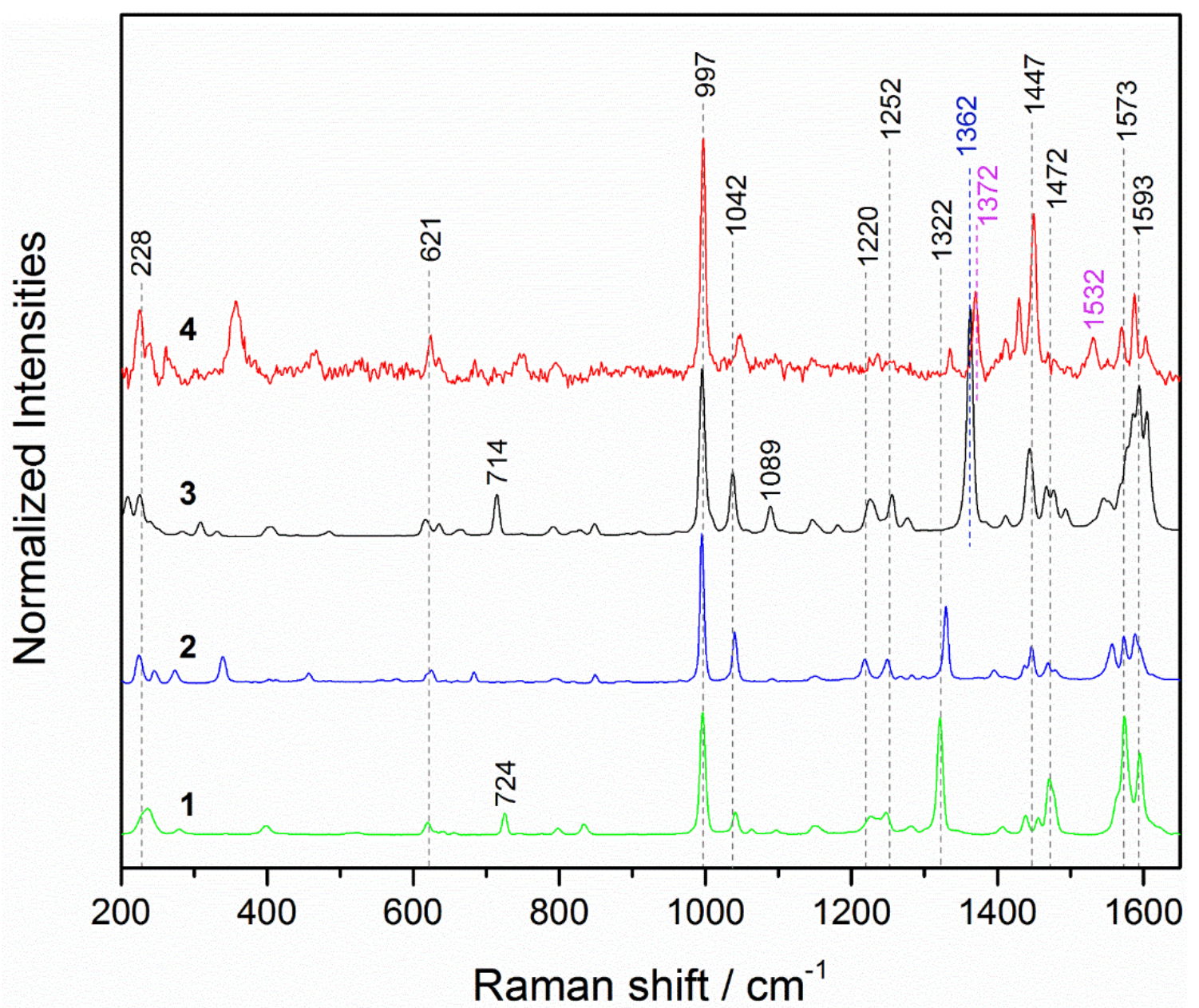

Figure S2: Off-resonance Raman spectra of ligands 1 - $4\left(\lambda_{\mathrm{ex}}=780 \mathrm{~nm}\right)$, solid state. 

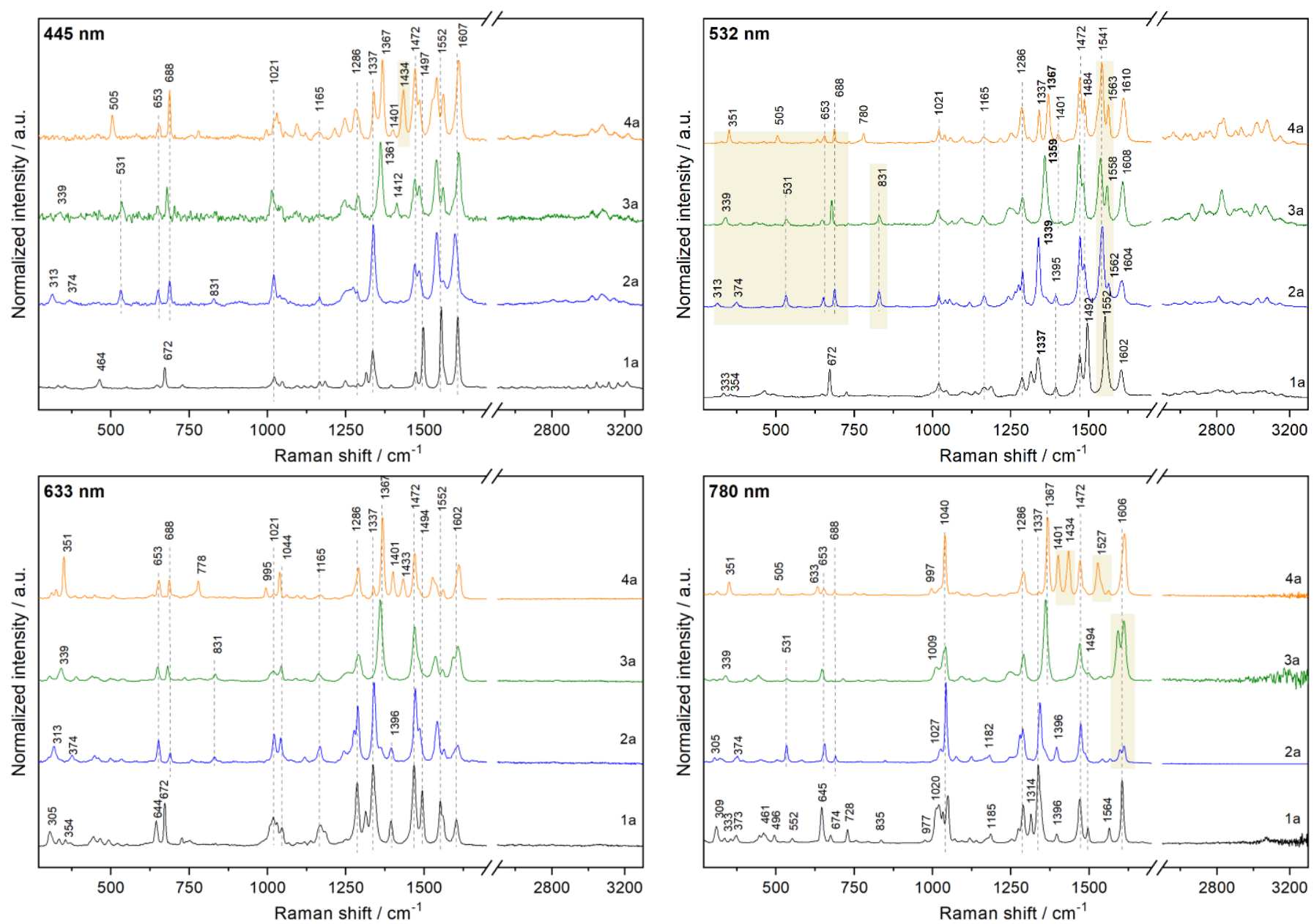

Figure S3 - Raman and resonance Raman spectra of complexes 1a-4a in solid state. Excitation wavelength are depicted in pictures. 


\section{A}

Transition \#8, $2.8713 \mathrm{eV}, f=0,0777$, symmetry $E$

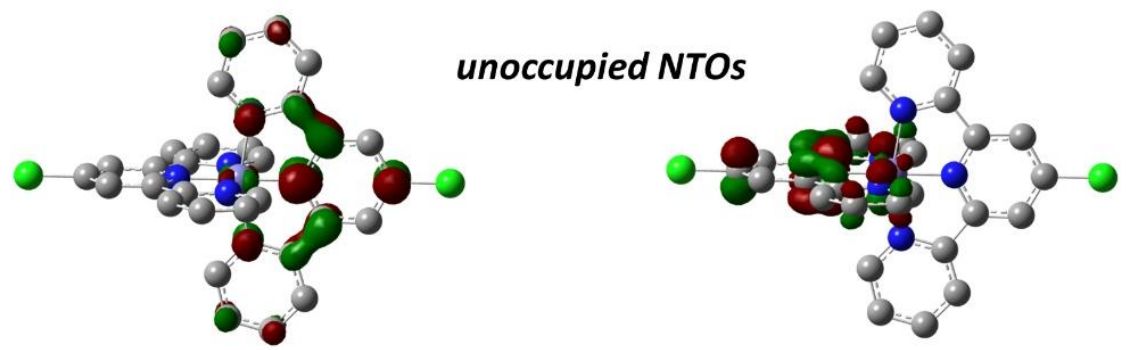

occupations $=0.46445$

0.46445

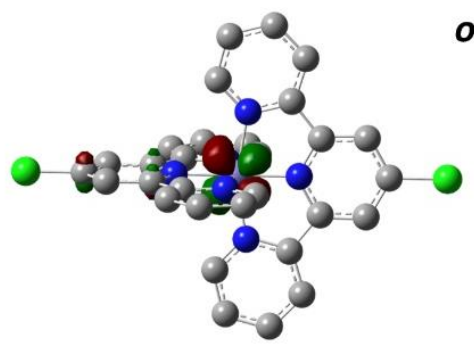

occupations $=0.46445$ occupied NTOs

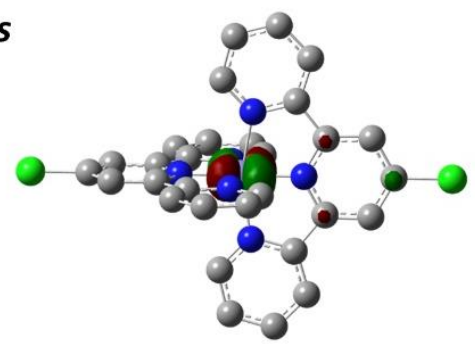

0.46445

B

Transition \#12, $3.0564 \mathrm{eV}, f=0,0669$, symmetry B2

occupations $=0.92999$

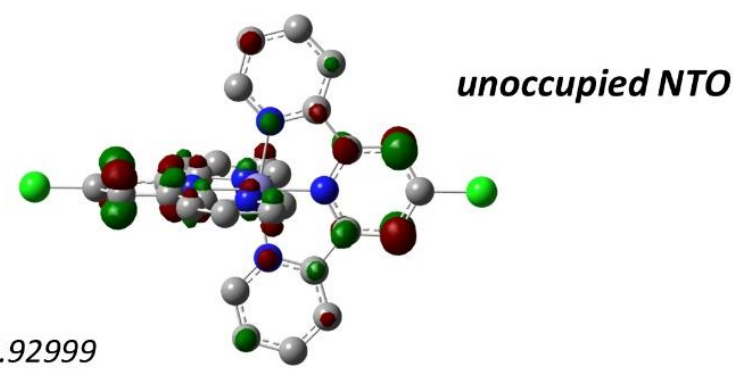

occupations $=0.92999$

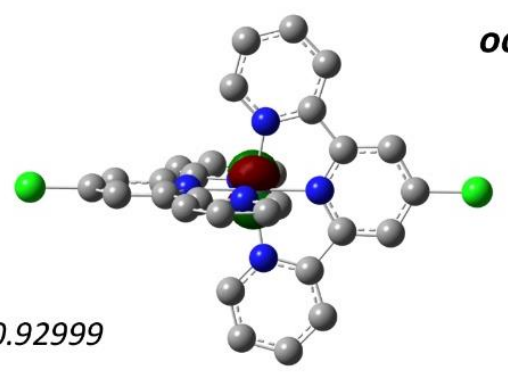

Figure S4 - Natural transition orbitals (NTOs) for $\mathbf{2 a}$ for transitions \#8 and \#12 (Table S3). 
A Transition \#6, $2.6479 \mathrm{eV}, f=0,5046$, symmetry B
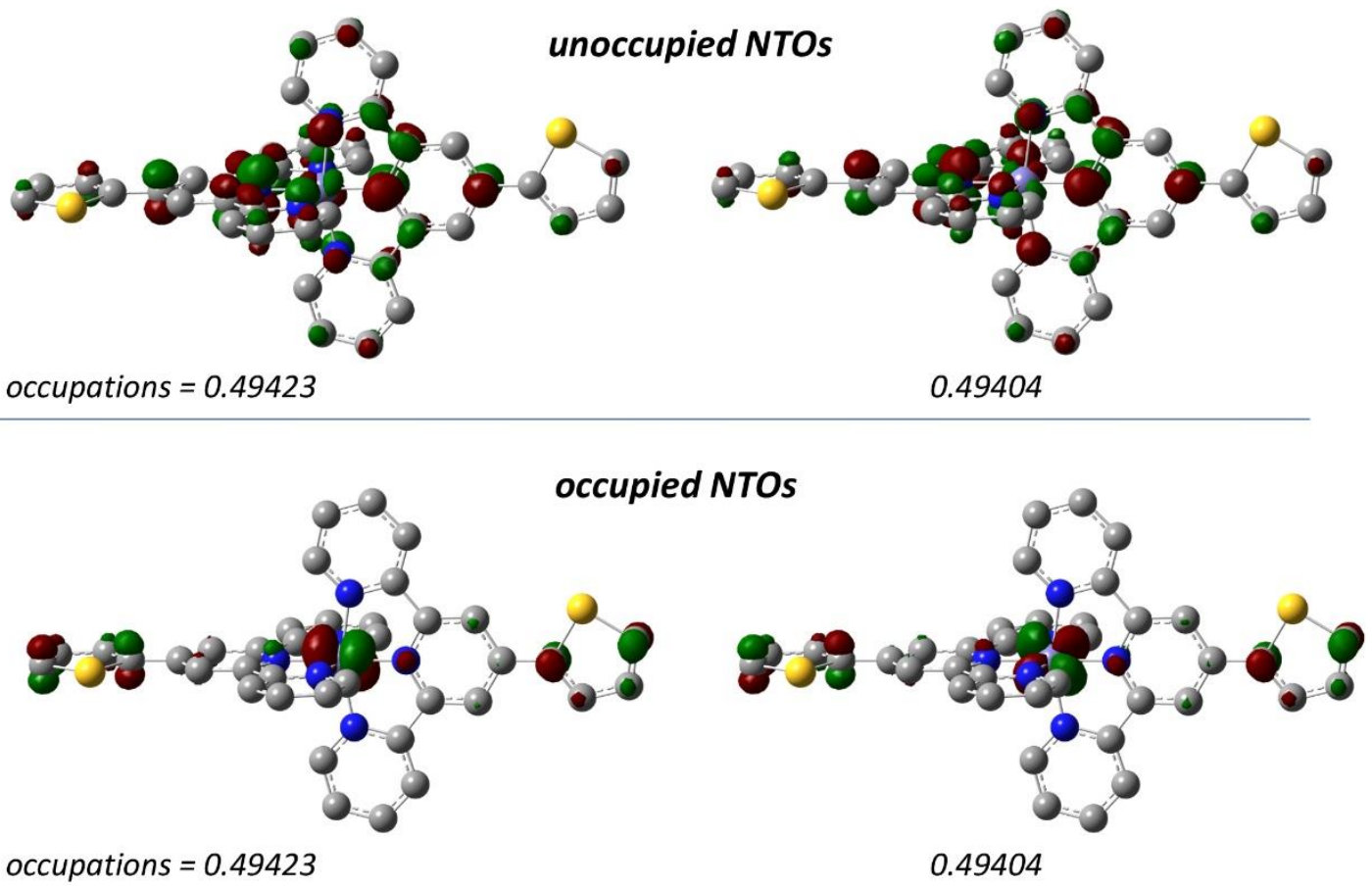

B Transition \#14, $3.0709 \mathrm{eV}, f=0,1057$, symmetry $B$

unoccupied NTO

occupations $=0.98207$

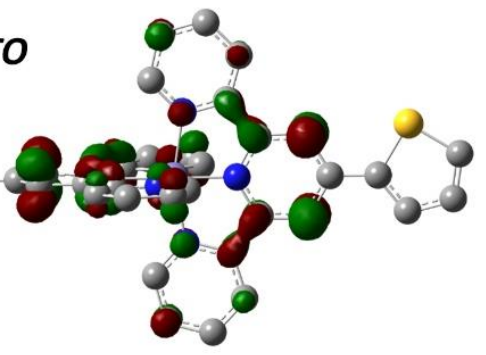

occupations $=0.98207$

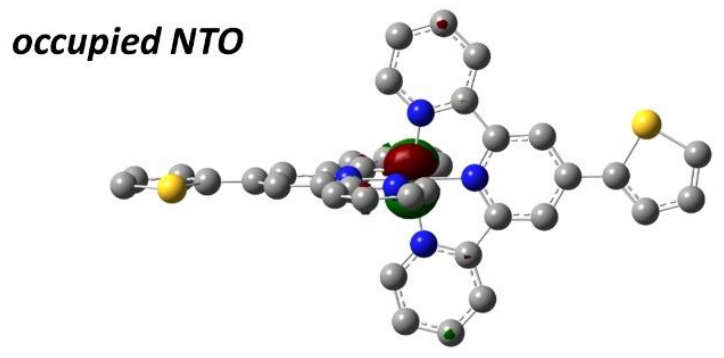

Figure S5 - Natural transition orbitals (NTOs) for 4a for transitions \#6 and \#14 (Table S4). 


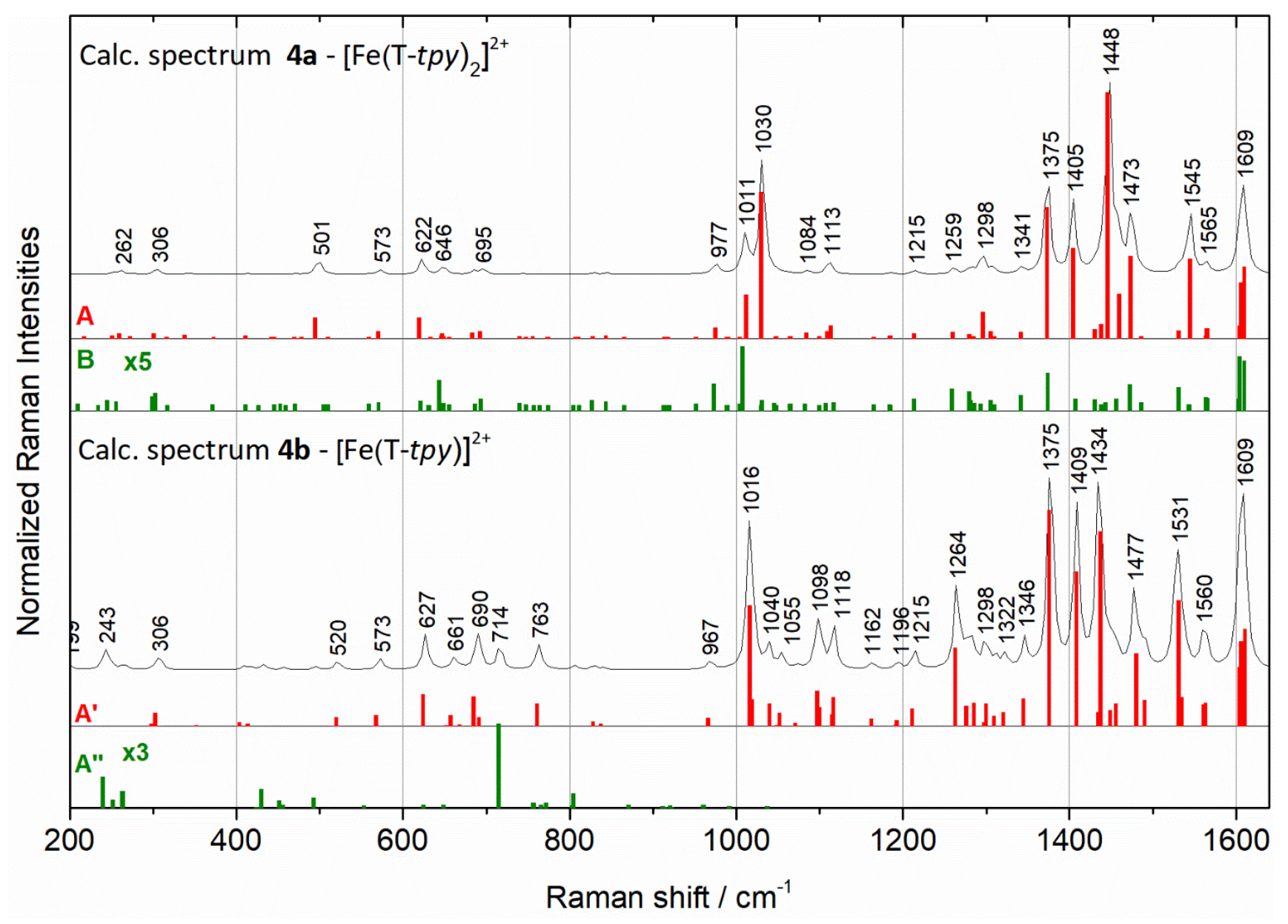

Figure $\mathbf{S 6}$ - Calculated spectra of $\mathbf{4 a}$ and $\mathbf{4 b}$ together with symmetry of normal modes. 


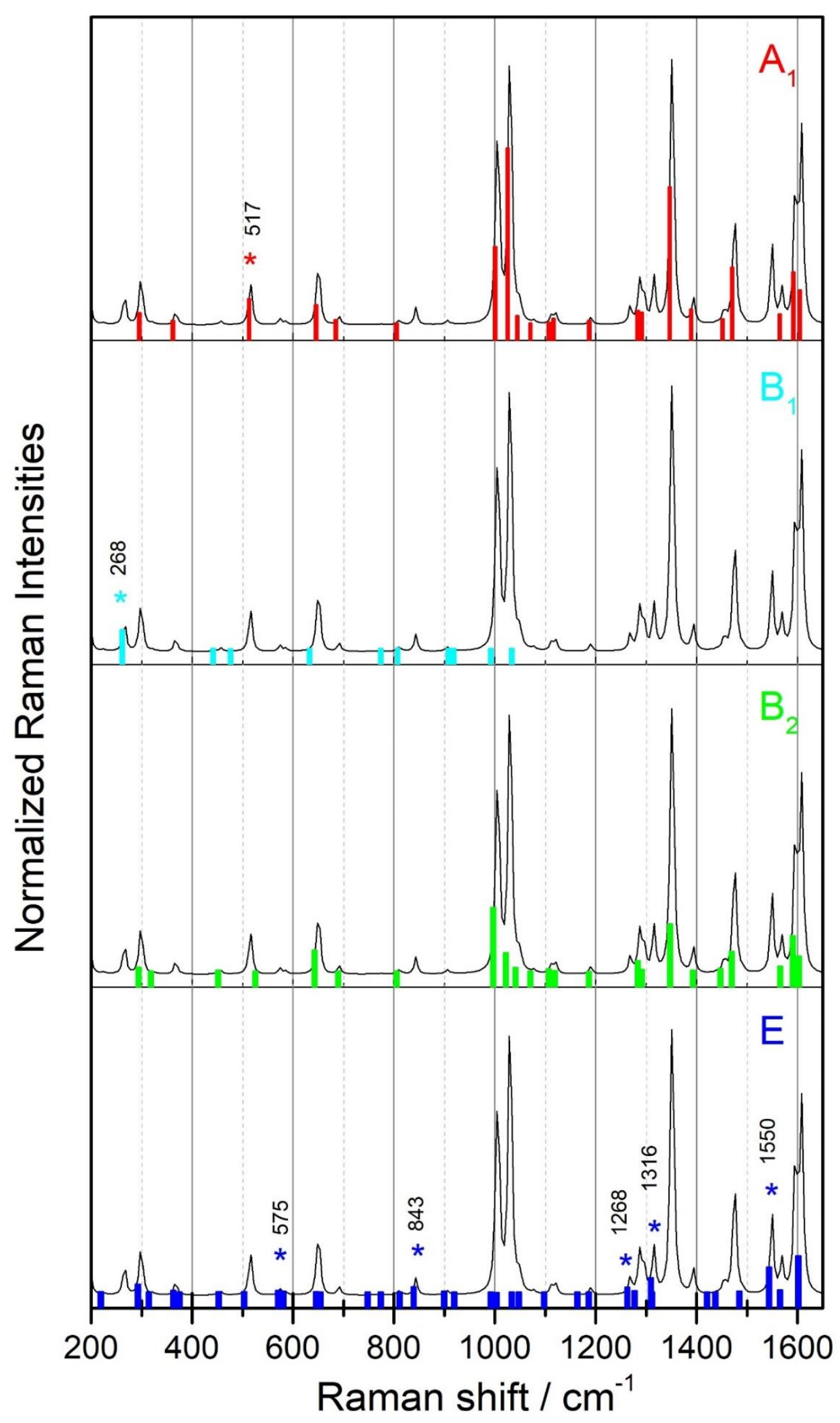

Figure S5 - Calculated spectra of $\mathbf{2 a}$ together with symmetry of normal modes. With asterisk are labeled bands consisting of modes of only one symmetry. 


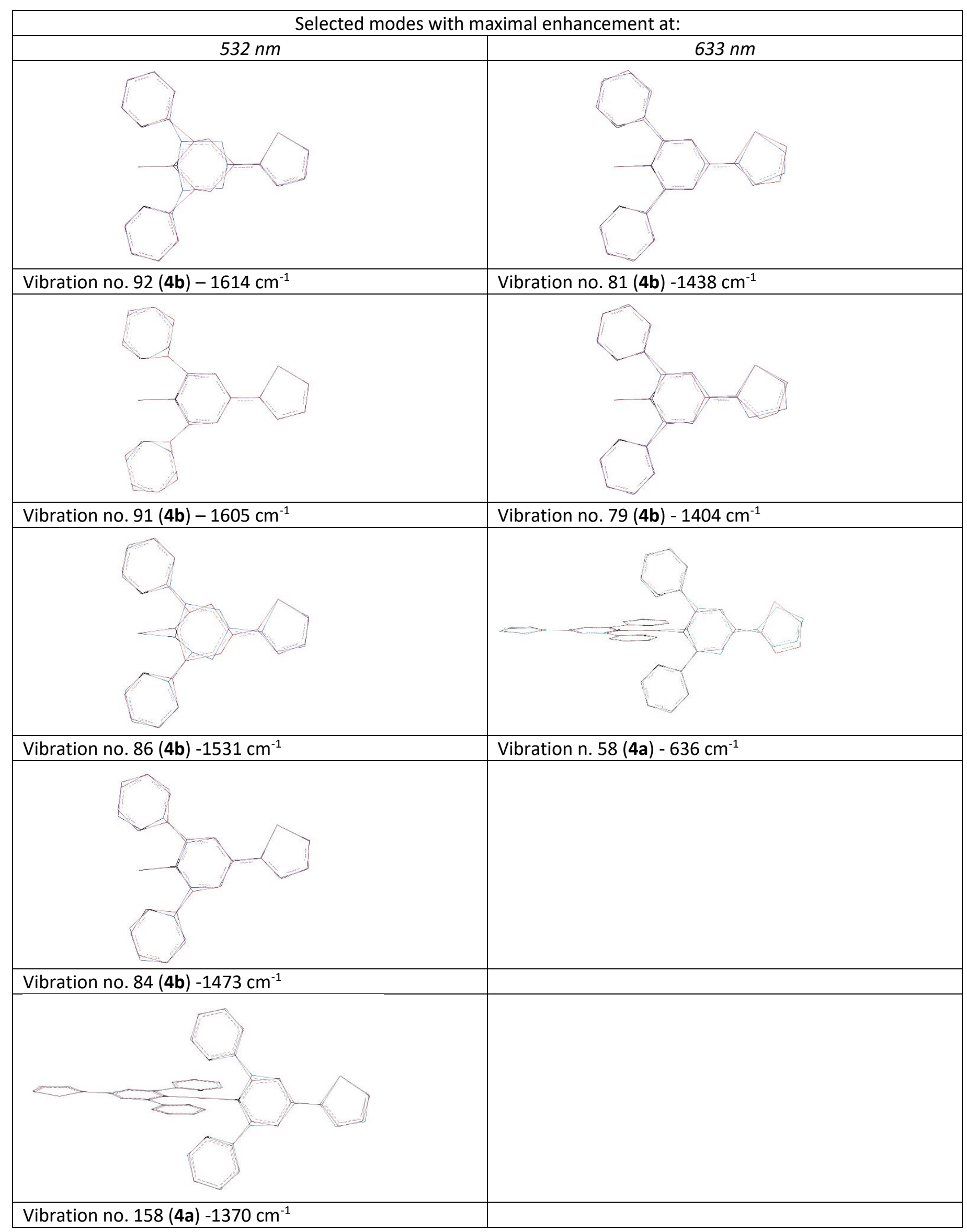

Figure S6 - Scheme of selected vibration modes with maximal enhancement at 532 and $633 \mathrm{~nm}$ excitations. 
Table S1: List of observed and calculated normal vibration modes and bands wavenumbers for $2 \mathrm{a}$ in the region $300-1650 \mathrm{~cm}^{-1}$.

\begin{tabular}{|c|c|c|c|c|c|c|c|}
\hline No. & Sym. & $\begin{array}{l}\text { Calc. } \\
\text { Raman }\end{array}$ & & Calc. & & Exp. & Vibrational mode assignment \\
\hline & & intensity & $\begin{array}{l}\text { non } \\
\text { scaled }\end{array}$ & $\begin{array}{c}\text { scaled } \\
f=0,975\end{array}$ & $\begin{array}{l}\text { spectral } \\
\text { band }\end{array}$ & $\begin{array}{l}785 \mathrm{~nm} \\
\text { excitation }\end{array}$ & \\
\hline 27 & $\mathrm{E}$ & 12 & 323 & 314 & \multirow{3}{*}{297} & \multirow{3}{*}{318} & \\
\hline 28 & $\mathrm{E}$ & 12 & 323 & 314 & & & $v(\mathrm{FeN})_{1}, \delta_{\text {in }}(\mathrm{CNFe})_{1}, \omega(\mathrm{NNNFe})_{1}, \tau(\mathrm{CNFeN}),(\mathrm{CCFeN})$ \\
\hline 29 & B2 & 10 & 326 & 318 & & & $v(\mathrm{FeN})_{\mathrm{c}}, \mathrm{v}(\mathrm{ClC}), \delta_{\text {in }}(\mathrm{CNC})_{\mathrm{c}}$ \\
\hline 30 & A1 & 117 & 371 & 362 & \multirow{3}{*}{366} & \multirow{3}{*}{370} & $\mathrm{v}(\mathrm{ClC}), \delta(\mathrm{CNFe})$ \\
\hline 31 & $\mathrm{E}$ & 62 & 372 & 363 & & & \\
\hline 32 & $E$ & 62 & 372 & 363 & & & $v(C C)_{c-1,}, O(C I C C), O(C C C)_{c-c-1}$ \\
\hline 33 & $\mathrm{E}$ & 5 & 385 & 375 & \multirow[b]{2}{*}{375} & & \multirow[b]{2}{*}{$\mathrm{v}(\mathrm{FeN})_{\mathrm{l}}, \delta(\mathrm{CCC})_{\mathrm{c-c-l}}$} \\
\hline 34 & $\mathrm{E}$ & 5 & 385 & 375 & & & \\
\hline 35 & A2 & 0 & 437 & 427 & & & $\tau(\text { CCNFe })_{1}$ \\
\hline 36 & B1 & 11 & 453 & 442 & \multirow{4}{*}{458} & \multirow{4}{*}{450} & $\tau(\mathrm{CCCN})_{1}, \tau(\mathrm{CCCN})_{\mathrm{C}}$ \\
\hline 37 & B2 & 33 & 463 & 451 & & & $\delta(\mathrm{NFeN})_{1}$ \\
\hline 38 & $\mathrm{E}$ & 20 & 464 & 453 & & & \multirow[b]{2}{*}{$\tau(\mathrm{CCCN})_{1}, \omega(\mathrm{NNNFe})_{1}, \omega(\mathrm{CFeCN})_{1}, \omega(\mathrm{CCFeN})_{1}$} \\
\hline 39 & $\mathrm{E}$ & 20 & 464 & 453 & & & \\
\hline 40 & $\mathrm{~A} 2$ & 0 & 471 & 459 & & & $\tau(\mathrm{CCCN})_{1,} \tau(\mathrm{CCCC})_{1-\mathrm{C}}$ \\
\hline 41 & B1 & 0 & 488 & 476 & & & $\tau(\mathrm{CCNFe}), \tau(\mathrm{CCNFe})$ \\
\hline 42 & $E$ & 15 & 516 & 503 & \multirow[b]{2}{*}{503} & \multirow{2}{*}{500} & \multirow{2}{*}{$\tau(\mathrm{CCCN})_{1,} \tau(\mathrm{CCNFe})_{1}, \omega(\mathrm{FeCCN})_{1}, \tau(\mathrm{CCCC})_{1-\mathrm{c}}$} \\
\hline 43 & $\mathrm{E}$ & 15 & 516 & 503 & & & \\
\hline 44 & A1 & 754 & 526 & 513 & \multirow{2}{*}{517} & \multirow{2}{*}{531} & $\mathrm{v}(\mathrm{ClC})$ \\
\hline 45 & B2 & 4 & 539 & 525 & & & $v(\mathrm{ClC}), \delta(\mathrm{NFeN})$ \\
\hline 46 & $E$ & 57 & 585 & 570 & \multirow{4}{*}{575} & \multirow{4}{*}{580} & \multirow[b]{2}{*}{$\delta(C C C)_{c-1}, \delta(N C C)_{c-1}, \delta(C I C C)$} \\
\hline 47 & $\mathrm{E}$ & 57 & 585 & 570 & & & \\
\hline 48 & $E$ & 29 & 596 & 581 & & & \multirow[b]{2}{*}{$\tau(\mathrm{HCCC})_{c}, \omega(\mathrm{ClCCC})_{c}$} \\
\hline 49 & $\mathrm{E}$ & 29 & 596 & 581 & & & \\
\hline 50 & $\mathrm{~A} 2$ & 0 & 647 & 631 & & & $\tau(\mathrm{CCCN})_{c}, \tau(\mathrm{CNFeN})_{l}$ \\
\hline 51 & B1 & 2 & 649 & 633 & 633 & & $\tau(\mathrm{HCCC})_{1}, \tau(\mathrm{CCCN})_{c}, \tau(\mathrm{CCCN})_{1}, \tau(\mathrm{CNFeN})_{1}$ \\
\hline
\end{tabular}




\begin{tabular}{|c|c|c|c|c|c|c|c|}
\hline No. & Sym. & $\begin{array}{l}\text { Calc. } \\
\text { Raman }\end{array}$ & & Calc. & & Exp. & Vibrational mode assignment \\
\hline & & intensity & $\begin{array}{c}\text { non } \\
\text { scaled }\end{array}$ & $\begin{array}{c}\text { scaled } \\
f=0,975\end{array}$ & $\begin{array}{l}\text { spectral } \\
\text { band }\end{array}$ & $\begin{array}{l}785 \mathrm{~nm} \\
\text { excitation }\end{array}$ & \\
\hline 52 & B2 & 611 & 659 & 643 & \multirow{6}{*}{648} & \multirow{6}{*}{651} & $\delta(\mathrm{CCN})_{1}, v_{s}(\mathrm{FeN})_{1}-$ in-plane - both tpy units \\
\hline 53 & $\mathrm{E}$ & 26 & 662 & 646 & & & \multirow[b]{2}{*}{$\delta(\mathrm{CCC})_{1}, \delta(\mathrm{CNC})_{1} \mathrm{v}_{\mathrm{as}}(\mathrm{FeN})_{1}$} \\
\hline 54 & $\mathrm{E}$ & 26 & 662 & 646 & & & \\
\hline 55 & A1 & 572 & 662 & 646 & & & $\delta(\mathrm{CCN})_{1}, v_{s}(\mathrm{FeN})_{1}-$ in-plane - both tpy units \\
\hline 56 & $\mathrm{E}$ & 1 & 672 & 655 & & & \multirow{2}{*}{$\begin{array}{l}\left.\delta(\mathrm{CCC})_{1}, \delta(\mathrm{CCC})_{c}, \delta(\mathrm{CCN})_{1}, \delta(\mathrm{NCC})_{c-1}, \delta(\mathrm{CCN})_{c}-\text { in plane rings (both } \mathrm{c}, 1\right) \\
\text { deformation }\end{array}$} \\
\hline 57 & $\mathrm{E}$ & 1 & 672 & 655 & & & \\
\hline 58 & $\mathrm{~A} 1$ & 144 & 702 & 685 & \multirow[b]{2}{*}{692} & \multirow[b]{2}{*}{688} & \multirow[b]{2}{*}{$\delta(\mathrm{CCC})_{1}, \delta(\mathrm{CCC})_{c}, \delta(\mathrm{CCN})_{1}, \delta(\mathrm{NCC})_{1}, \delta(\mathrm{CNC})_{c}, v(\mathrm{CCl})$ - in plane } \\
\hline 59 & B2 & 9 & 707 & 689 & & & \\
\hline 60 & $\mathrm{E}$ & 4 & 767 & 748 & \multirow[b]{2}{*}{748} & & \multirow[b]{2}{*}{$\tau(\mathrm{HCCN})_{c, l}, \omega(\mathrm{CCFeN})_{c, l}, \omega(\mathrm{ClCCC})_{c}, \omega(\mathrm{CNCC})_{c, l}$ - out of plane } \\
\hline 61 & $\mathrm{E}$ & 4 & 767 & 748 & & & \\
\hline 62 & $\mathrm{~A} 2$ & 0 & 783 & 763 & & & $\tau(\mathrm{HCCN})_{1}, \tau(\mathrm{HCCC})_{c}-$ out of plane \\
\hline 63 & $\mathrm{E}$ & 4 & 793 & 774 & \multirow{3}{*}{774} & & \multirow[b]{2}{*}{$\tau(\mathrm{HCCN})_{\mathrm{c}, \mathrm{I}}$ - out of plane } \\
\hline 64 & $\mathrm{E}$ & 4 & 793 & 774 & & & \\
\hline 65 & B1 & 2 & 794 & 774 & & & $\tau(\mathrm{HCCN})_{c, 1}-$ out of plane \\
\hline 66 & A1 & 50 & 826 & 805 & \multirow{6}{*}{809} & & $v(\mathrm{CCl}), \delta(\mathrm{CCC})_{c}, \delta(\mathrm{CNC})_{c}-$ in plane \\
\hline 67 & $\mathrm{~A} 2$ & 0 & 826 & 805 & & & $\tau(\mathrm{HCCC})_{c, 1}-$ out of plane \\
\hline 68 & B2 & 13 & 826 & 805 & & & $v(\mathrm{CCl}), \delta(\mathrm{CCC})_{c}, \delta(\mathrm{CNC})_{c}-$ in plane \\
\hline 69 & B1 & 20 & 828 & 807 & & & $\tau(\mathrm{HCCC})_{c, 1}-$ out of plane \\
\hline 70 & $\mathrm{E}$ & 4 & 832 & 811 & & & \multirow[b]{2}{*}{$\tau(\mathrm{HCCC})_{c, l}, \tau(\mathrm{HCCN})_{c, l}, \omega(\mathrm{CCFeN})_{c}-$ out of plane } \\
\hline 71 & $\mathrm{E}$ & 4 & 832 & 811 & & & \\
\hline 72 & $\mathrm{E}$ & 153 & 861 & 839 & \multirow{2}{*}{839} & \multirow{2}{*}{844} & \multirow{2}{*}{$\delta(\mathrm{CCC})_{c, l}, \delta(\mathrm{CNC})_{c, l}, \delta(\mathrm{CCN})_{c, 1}-$ in plane } \\
\hline 73 & $\mathrm{E}$ & 153 & 861 & 839 & & & \\
\hline 74 & $\mathrm{E}$ & 36 & 923 & 900 & \multirow{4}{*}{907} & & \multirow{2}{*}{$\tau(\mathrm{HCCN})_{c}, \omega(\mathrm{ClCCC})_{c}, \tau(\mathrm{CCCN})_{c}$ - out of plane (H on the $c$ units) } \\
\hline 75 & $\mathrm{E}$ & 36 & 923 & 900 & & & \\
\hline 76 & $\mathrm{~A} 2$ & 0 & 930 & 907 & & & $\tau(\mathrm{HCCC})_{c}$ - out of plane (H on the $c$ units) \\
\hline 77 & B1 & 10 & 931 & 908 & & & $\tau(\mathrm{HCCN})_{c}-$ out of plane (H on the $\mathrm{c}$ units) \\
\hline
\end{tabular}




\begin{tabular}{|c|c|c|c|c|c|c|c|}
\hline No. & Sym. & $\begin{array}{l}\text { Calc. } \\
\text { Raman }\end{array}$ & & Calc. & & Exp. & Vibrational mode assignment \\
\hline & & intensity & $\begin{array}{l}\text { non } \\
\text { scaled }\end{array}$ & $\begin{array}{c}\text { scaled } \\
f=0,975\end{array}$ & $\begin{array}{l}\text { spectral } \\
\text { band }\end{array}$ & $\begin{array}{l}785 \mathrm{~nm} \\
\text { excitation }\end{array}$ & \\
\hline 78 & B1 & 2 & 942 & 918 & \multirow{3}{*}{918} & & $\tau(\mathrm{HCCN})_{1}-$ out of plane (H on the I units) \\
\hline 79 & $\mathrm{E}$ & 2 & 943 & 919 & & & \multirow{2}{*}{$\tau(\mathrm{HCCC})_{1}, \tau(\mathrm{HCCN})_{1}-$ out of plane (H on the I units) } \\
\hline 80 & $\mathrm{E}$ & 2 & 943 & 919 & & & \\
\hline 81 & A2 & 0 & 944 & 920 & & & $\tau(\mathrm{HCCN})_{c, 1}-$ out of plane \\
\hline 82 & A2 & 0 & 1017 & 992 & \multirow{4}{*}{992} & \multirow{4}{*}{981} & $\tau(\mathrm{HCCN})_{1}-$ out of plane (H on the I units) \\
\hline 83 & $E$ & 2 & 1018 & 992 & & & \\
\hline 84 & $\mathrm{E}$ & 2 & 1018 & 992 & & & $\tau(\mathrm{HCCC})_{1}-$ out of plane (H on the I units) \\
\hline 85 & B1 & 3 & 1018 & 992 & & & $\tau(\mathrm{HCCN})_{\mid}$- out of plane (H on the I units) \\
\hline 86 & B2 & 1848 & 1022 & 997 & \multirow{2}{*}{1004} & \multirow{2}{*}{1021} & $v_{s}(\mathrm{FeN})_{1}, v(N C)_{1}, v(C C)_{1}, \delta(C C N)_{1}-$ breathing vibration I units \\
\hline 87 & $\mathrm{~A} 1$ & 2258 & 1026 & 1001 & & & $\mathrm{v}_{s}(\mathrm{FeN})_{1}, \mathrm{v}(\mathrm{NC})_{1}, \mathrm{v}(\mathrm{CC})_{1}, \delta(\mathrm{CCN})_{1}-$ breathing vibration I units \\
\hline 88 & $E$ & 0 & 1029 & 1003 & & & \multirow{2}{*}{$v_{\text {as }}(\mathrm{FeN})_{1} \delta(\mathrm{CNC})_{1}, \delta(\mathrm{CCN})_{1}, \delta(\mathrm{CCC})_{1}$ - triangular breathing vibration I units } \\
\hline 89 & $E$ & 0 & 1029 & 1003 & & & \\
\hline 90 & B2 & 541 & 1048 & 1022 & \multirow{6}{*}{1029} & \multirow{6}{*}{1043} & $\mathrm{v}_{\mathrm{as}}(\mathrm{FeN})_{c}, \delta(\mathrm{CNC})_{c}, \delta(\mathrm{CCN})_{c}, \delta(\mathrm{CCC})_{c}$ - triangular breathing vibration $\mathrm{c}$ units \\
\hline 91 & A1 & 5117 & 1052 & 1026 & & & $v_{s}(\mathrm{FeN})_{c}, \delta(\mathrm{CNC})_{c}, \delta(\mathrm{CCN})_{c}, \delta(\mathrm{CCC})_{c}-$ triangular breathing vibration $\mathrm{c}$ units \\
\hline 92 & E & 2 & 1060 & 1034 & & & \\
\hline 93 & $\mathrm{E}$ & 2 & 1060 & 1034 & & & $\tau(\mathrm{HCCC})_{1}, \tau(\mathrm{HCCN})_{1}, \tau(\mathrm{CCCN})_{1}-$ out of plane (H on the I units) \\
\hline 94 & B1 & 8 & 1060 & 1034 & & & $\tau(\mathrm{HCCN})_{1}-$ out of plane (H on the I units) \\
\hline 95 & A2 & 0 & 1060 & 1034 & & & $\tau(\mathrm{HCCC})_{1}-$ out of plane ( $\mathrm{H}$ on the I units) \\
\hline 96 & B2 & 103 & 1068 & 1041 & \multirow{4}{*}{1043} & \multirow{4}{*}{1055} & $v(C C)_{1}, \delta(C N C)_{1}, \delta(C C C)_{c}-$ in plane \\
\hline 97 & A1 & 264 & 1071 & 1044 & & & $v(C C)_{1}, \delta(C N C)_{1}, \delta(\mathrm{HCC})_{1}-$ in plane \\
\hline 98 & $\mathrm{E}$ & 2 & 1074 & 1047 & & & \\
\hline 99 & $\mathrm{E}$ & 2 & 1074 & 1047 & & & $v(C C), 0(H C C),-$ In plane (I units) \\
\hline 100 & B2 & 4 & 1098 & 1070 & \multirow{2}{*}{1077} & \multirow{2}{*}{1074} & $\delta(\mathrm{HCC})_{1, \mathrm{c}}-$ in plane \\
\hline 101 & A1 & 49 & 1098 & 1071 & & & $\delta(\mathrm{CCN})_{l, c}, \delta(\mathrm{HCC})_{l, c}-$ in plane \\
\hline 102 & E & 2 & 1126 & 1098 & \multirow{4}{*}{1111} & \multirow{4}{*}{1114} & \multirow{2}{*}{$\delta(\mathrm{HCC})_{1, c}, \delta(\mathrm{CNC})_{1}, \mathrm{v}(\mathrm{CC})_{\mathrm{c}}$ - in plane } \\
\hline 103 & $\mathrm{E}$ & 2 & 1126 & 1098 & & & \\
\hline 104 & B2 & 76 & 1135 & 1106 & & & $\delta(\mathrm{HCC})_{c}, \delta(\mathrm{HCC})_{1}-$ in plane (mainly $\mathrm{H}$ on the $c$ units) \\
\hline 105 & A1 & 67 & 1136 & 1107 & & & $\delta(\mathrm{HCC})_{c}, \delta(\mathrm{HCC})_{1}-$ in plane (mainly $\mathrm{H}$ on the $\mathrm{c}$ units) \\
\hline
\end{tabular}




\begin{tabular}{|c|c|c|c|c|c|c|c|}
\hline No. & Sym. & $\begin{array}{l}\text { Calc. } \\
\text { Raman }\end{array}$ & & Calc. & & Exp. & Vibrational mode assignment \\
\hline & & intensity & $\begin{array}{c}\text { non } \\
\text { scaled }\end{array}$ & $\begin{array}{c}\text { scaled } \\
f=0,975\end{array}$ & $\begin{array}{l}\text { spectral } \\
\text { band }\end{array}$ & $\begin{array}{l}785 \mathrm{~nm} \\
\text { excitation }\end{array}$ & \\
\hline 106 & A1 & 186 & 1145 & 1116 & \multirow{2}{*}{1121} & \multirow{2}{*}{1120} & $\delta(\mathrm{HCC})_{c}, \delta(\mathrm{HCC})_{1}-$ in plane \\
\hline 107 & B2 & 14 & 1148 & 1119 & & & $\delta(\mathrm{HCC})_{c}, \delta(\mathrm{HCC})_{1}-$ in plane \\
\hline 108 & $\mathrm{E}$ & 1 & 1193 & 1163 & \multirow{2}{*}{1163} & & \multirow{2}{*}{$\delta(\mathrm{HCC})_{c}, \delta(\mathrm{HCC})_{1}-$ in plane } \\
\hline 109 & $\mathrm{E}$ & 1 & 1193 & 1163 & & & \\
\hline 110 & $\mathrm{E}$ & 6 & 1216 & 1186 & \multirow{4}{*}{1190} & \multirow{4}{*}{1165} & \multirow{2}{*}{$\delta(\mathrm{HCC})_{1}$ - in plane ( $\mathrm{H}$ on the I units) } \\
\hline 111 & $\mathrm{E}$ & 6 & 1216 & 1186 & & & \\
\hline 112 & B2 & 0 & 1217 & 1186 & & & $\delta(\mathrm{HCC})_{1}-$ in plane ( $\mathrm{H}$ on the I units) \\
\hline 113 & A1 & 120 & 1217 & 1187 & & & $\delta(\mathrm{HCC})_{1}-$ in plane ( $\mathrm{H}$ on the I units) \\
\hline 114 & E & 150 & 1295 & 1263 & \multirow[b]{2}{*}{1268} & \multirow[b]{2}{*}{1244} & \multirow[b]{2}{*}{$\delta(\mathrm{HCC})_{c}-$ in plane ( $\mathrm{H}$ on the $c$ units), $v(\mathrm{CC})_{c-1}$} \\
\hline 115 & $\mathrm{E}$ & 150 & 1295 & 1263 & & & \\
\hline 116 & $\mathrm{E}$ & 45 & 1310 & 1277 & \multirow{6}{*}{1287} & \multirow{6}{*}{1275} & \multirow[b]{2}{*}{$v(\mathrm{NC})_{c, l}, v(\mathrm{CC})_{c, l}, \delta(\mathrm{HCN})_{1}-$ in plane } \\
\hline 117 & $\mathrm{E}$ & 45 & 1310 & 1277 & & & \\
\hline 118 & A1 & 414 & 1316 & 1283 & & & $v(N C)_{1}, v(C C)_{1}-$ mainly on the I units, in plane \\
\hline 119 & B2 & 310 & 1316 & 1283 & & & $v(N C)_{1, c}, v(C C)_{1}-$ in plane \\
\hline 120 & B2 & 60 & 1324 & 1291 & & & $v(\mathrm{NC})_{1}, \delta(\mathrm{HCC})_{1}, \delta(\mathrm{HCN})_{1}-$ mainly on the I units - in plane \\
\hline 121 & A1 & 363 & 1324 & 1291 & & & $v(C C)_{1}, \delta(H C C)_{1}-$ mainly on the I units - in plane \\
\hline 122 & E & 421 & 1343 & 1309 & \multirow{4}{*}{1316} & \multirow{4}{*}{1288} & \multirow[b]{2}{*}{$v(\mathrm{NC})_{c, l}, v(\mathrm{CC})_{c}-$ mainly on the $c$ units, $\delta(\mathrm{HCC})_{1}-$ in plane } \\
\hline 123 & E & 421 & 1343 & 1309 & & & \\
\hline 124 & E & 8 & 1345 & 1311 & & & \multirow[b]{2}{*}{$v(C C)_{1}, \delta(H C C)_{1}-$ mainly on the I units, in plane } \\
\hline 125 & $\mathrm{E}$ & 8 & 1345 & 1311 & & & \\
\hline 126 & A1 & 3975 & 1381 & 1347 & \multirow{2}{*}{1350} & \multirow{2}{*}{1342} & \multirow{2}{*}{$v(\mathrm{CCl}), v(\mathrm{CC})_{c-1}, \delta(\mathrm{CNC})_{c}, \delta(\mathrm{CCC})_{c}, \delta(\mathrm{HCC})_{c, 1}-$ in plane } \\
\hline 127 & B2 & 1368 & 1382 & 1348 & & & \\
\hline 128 & A1 & 444 & 1425 & 1389 & \multirow{2}{*}{1394} & \multirow{2}{*}{1396} & $v(\mathrm{CN})_{c}, v(\mathrm{CC})_{c}, \delta(\mathrm{HCC})_{c, 1}, \delta(\mathrm{NCC})_{c-1}-$ in plane \\
\hline 129 & B2 & 32 & 1428 & 1392 & & & $v(C N)_{c}, v(C C)_{c}, \delta(H C C)_{c, 1}, \delta(N C C)_{c-1}-$ in plane \\
\hline 130 & $\mathrm{E}$ & 1 & 1457 & 1421 & & & \multirow{2}{*}{$v(\mathrm{CC})_{c, 1}, v(\mathrm{CC})_{\mathrm{c}-1,} \delta(\mathrm{HCC})_{c, 1}$} \\
\hline 131 & $\mathrm{E}$ & 1 & 1457 & 1421 & & & \\
\hline
\end{tabular}




\begin{tabular}{|c|c|c|c|c|c|c|c|}
\hline No. & Sym. & $\begin{array}{l}\text { Calc. } \\
\text { Raman }\end{array}$ & & Calc. & & Exp. & Vibrational mode assignment \\
\hline & & intensity & $\begin{array}{c}\text { non } \\
\text { scaled }\end{array}$ & $\begin{array}{c}\text { scaled } \\
f=0,975\end{array}$ & $\begin{array}{l}\text { spectral } \\
\text { band }\end{array}$ & $\begin{array}{c}785 \mathrm{~nm} \\
\text { excitation }\end{array}$ & \\
\hline 132 & $\mathrm{E}$ & 9 & 1474 & 1437 & \multirow{4}{*}{1453} & \multirow{4}{*}{1453} & \\
\hline 133 & $\mathrm{E}$ & 9 & 1474 & 1437 & & & $\delta(\mathrm{HCC})_{1}, \delta(\mathrm{NCC})_{\mathrm{C}-1}-$ in plane \\
\hline 134 & B2 & 88 & 1484 & 1447 & & & $v(\mathrm{CN})_{c, 1}, \delta(\mathrm{HCC})_{c, 1}-$ in plane \\
\hline 135 & A1 & 170 & 1489 & 1452 & & & $\mathrm{v}(\mathrm{CN})_{c, 1}, \delta(\mathrm{HCC})_{c, 1}-$ in plane \\
\hline 136 & B2 & 578 & 1507 & 1469 & \multirow{2}{*}{1477} & \multirow{2}{*}{1472} & $\mathrm{v}(\mathrm{CC})_{\mathrm{C}-1}, \delta(\mathrm{HCC})_{1}-$ in plane \\
\hline 137 & A1 & 1659 & 1508 & 1470 & & & $v(C C)_{c-1}, \delta(H C C)_{1}-$ in plane \\
\hline 138 & $\mathrm{E}$ & 27 & 1523 & 1485 & \multirow[b]{2}{*}{1485} & \multirow[b]{2}{*}{1485} & \multirow{2}{*}{$v(C C)_{c-1}, v(C C)_{c, 1} \delta(H C C)_{c, 1}-$ in plane } \\
\hline 139 & $E$ & 27 & 1523 & 1485 & & & \\
\hline 140 & $\mathrm{E}$ & 725 & 1583 & 1543 & \multirow[b]{2}{*}{1550} & \multirow[b]{2}{*}{1540} & \multirow[b]{2}{*}{$v(N C)_{c}, v(C C)_{c}-$ in plane } \\
\hline 141 & $\mathrm{E}$ & 725 & 1583 & 1543 & & & \\
\hline 142 & A1 & 311 & 1605 & 1565 & \multirow{4}{*}{1570} & \multirow{4}{*}{1565} & $v(N C)_{1}, v(C C)_{1}, \delta(H C C)_{1}-$ in plane \\
\hline 143 & $\mathrm{E}$ & 75 & 1605 & 1565 & & & \\
\hline 144 & $\mathrm{E}$ & 75 & 1605 & 1565 & & & $v(N C)_{1}, v(C C)_{1}, \delta(H C C)_{1}$ - in plane \\
\hline 145 & B2 & 141 & 1605 & 1565 & & & $v(N C)_{1}, v(C C)_{1}, \delta(H C C)_{1}-$ in plane \\
\hline 146 & B2 & 1025 & 1632 & 1591 & \multirow{2}{*}{1594} & \multirow{2}{*}{1602} & $v(\mathrm{CC})_{c}, \delta(\mathrm{CNC})_{c}, \delta(\mathrm{CCC})_{c}, \delta(\mathrm{HCC})_{c}-$ in plane \\
\hline 147 & A1 & 1509 & 1632 & 1591 & & & $v(\mathrm{CC})_{c}, \delta(\mathrm{CNC})_{c}, \delta(\mathrm{CCC})_{c}, \delta(\mathrm{HCC})_{c}-$ in plane \\
\hline 148 & $\mathrm{E}$ & 1055 & 1643 & 1601 & \multirow{4}{*}{1609} & \multirow{4}{*}{1610} & \multirow[b]{2}{*}{$v(C C)_{1}, \delta(C N C)_{1}, \delta(C C C)_{1}, \delta(\mathrm{HCC})_{1}-$ in plane } \\
\hline 149 & $\mathrm{E}$ & 1055 & 1643 & 1601 & & & \\
\hline 150 & B2 & 437 & 1644 & 1603 & & & $v(\mathrm{CC})_{1}, \delta(\mathrm{CNC})_{1}, \delta(\mathrm{CCC})_{1}, \delta(\mathrm{HCC})_{1}-$ in plane \\
\hline 151 & A1 & 1003 & 1646 & 1604 & & & $v(C C)_{1}, \delta(C N C)_{1}, \delta(C C C)_{1}, \delta(H C C)_{1}-$ in plane \\
\hline
\end{tabular}

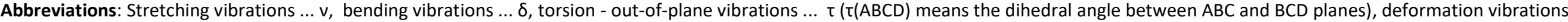

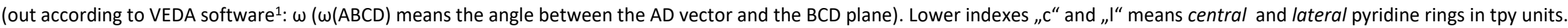

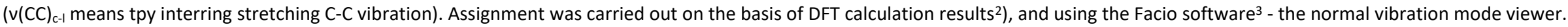


Table S2: List of observed vibration bands in $4 \mathbf{a}$ and calculated normal vibration modes of $4 \mathrm{a}\left(300-1375 \mathrm{~cm}^{-1}\right)$ and $4 \mathrm{~b}\left(300-1650 \mathrm{~cm}^{-1}\right)$.

\begin{tabular}{|c|c|c|c|c|c|c|c|c|c|c|c|c|}
\hline \multicolumn{5}{|c|}{$4 a$} & \multicolumn{5}{|c|}{ 4b } & \multirow{3}{*}{$\begin{array}{c}\text { Calc. } \\
\text { spectral } \\
\text { band }\end{array}$} & \multirow{3}{*}{$\begin{array}{c}\text { Exp.785 } \\
\text { nm } \\
\text { excit. }\end{array}$} & \multirow{3}{*}{ Vibrational mode assignment } \\
\hline \multirow[t]{2}{*}{ No. } & \multirow[t]{2}{*}{ Sym. } & \multirow{2}{*}{$\begin{array}{l}\text { Calc. } \\
\text { Raman } \\
\text { inten. }\end{array}$} & \multicolumn{2}{|c|}{ Calc. wavenumbers } & \multirow[t]{2}{*}{ No. } & \multirow[t]{2}{*}{ Sym. } & \multirow{2}{*}{$\begin{array}{c}\text { Calc. } \\
\text { Raman } \\
\text { inten. }\end{array}$} & \multicolumn{2}{|c|}{ Calc. wavenumbers } & & & \\
\hline & & & $\begin{array}{l}\text { non } \\
\text { scaled }\end{array}$ & $\begin{array}{c}\text { scaled } \\
f=0,975\end{array}$ & & & & $\begin{array}{l}\text { non } \\
\text { scaled }\end{array}$ & $\begin{array}{c}\text { scaled } \\
f=0,972\end{array}$ & & & \\
\hline 32 & A & 446 & 308 & 300 & & & & & & \multirow{2}{*}{306} & \multirow{2}{*}{310} & \multirow{2}{*}{$\delta(\mathrm{NFeN}), \omega(\mathrm{CCFeN})_{c}, \tau(\mathrm{CCCN})_{1}-$ out of pl. } \\
\hline 33 & $\mathrm{~B}$ & 315 & 310 & 302 & & & & & & & & \\
\hline 34 & A & 2 & 324 & 316 & & & & & & & & \multirow{2}{*}{$\delta(\mathrm{NFeN}), \omega(\mathrm{CCFeN})_{c}-$ out of pl. } \\
\hline 35 & $\mathrm{~B}$ & 1 & 325 & 317 & & & & & & & & \\
\hline 36 & $A$ & 248 & 346 & 337 & & & & & & 345 & 351 & $\begin{array}{l}v_{s}(\mathrm{FeN})_{c, l}, \delta(\mathrm{CNFe})_{1} \text { in-ph.- breathin of the Fe } \\
\text { center, rotation of I units - in pl. }\end{array}$ \\
\hline 37 & $\mathrm{~B}$ & 16 & 381 & 371 & & & & & & & & \multirow[b]{2}{*}{$v_{\text {as }}(\mathrm{FeN})_{\mathrm{l}}, \omega(\mathrm{CCFeN})_{\mathrm{c}}-$ out of $\mathrm{pl}$} \\
\hline 38 & A & 20 & 382 & 372 & & & & & & & & \\
\hline 39 & $\mathrm{~B}$ & 18 & 421 & 410 & & & & & & \multirow{2}{*}{413} & \multirow{2}{*}{415} & \multirow{2}{*}{$\delta(C C C)_{C-T}, \delta(C C C)_{C-1}-$ in pl. } \\
\hline 40 & A & 157 & 421 & 410 & & & & & & & & \\
\hline 41 & $B$ & 0 & 437 & 426 & & & & & & & & $\tau(\mathrm{CCCN})_{1}, \omega(\mathrm{CCFeN})_{1}-$ out of $\mathrm{pl}$ \\
\hline 42 & A & 8 & 453 & 442 & & & & & & & & $\tau(\mathrm{CCNFe})_{1}, \omega(\mathrm{CCNC})_{\mathrm{c}-1}-$ out of $\mathrm{pl}$ \\
\hline 43 & $B$ & 8 & 457 & 446 & & & & & & & & \multirow{2}{*}{$\tau(\mathrm{CCCS})_{T}, \tau(\mathrm{CCCC})_{\mathrm{C}-\mathrm{T}}, \tau(\mathrm{CCCN})_{\mathrm{C}, \mathrm{I}}-$ out of pl. } \\
\hline 44 & A & 11 & 457 & 446 & & & & & & & & \\
\hline 45 & $B$ & 44 & 464 & 452 & & & & & & & & $v_{\text {as }}(\mathrm{FeN})_{c}, \delta(\mathrm{CNFe})_{l}, \delta(\mathrm{NFeN})-$ in pl. \\
\hline 46 & B & 0 & 471 & 459 & & & & & & & & $\omega\left(\mathrm{CCNC}_{\mathrm{C}-\mathrm{I}}-\right.$ out of $\mathrm{pl}$ \\
\hline 47 & A & 37 & 481 & 469 & & & & & & & & \multirow{2}{*}{$\tau(\mathrm{CCCS})_{T}, \omega(\mathrm{CCFeN})_{1}, \omega(\mathrm{CCCC})_{1}-$ out of $\mathrm{pl}$} \\
\hline 48 & $\mathrm{~B}$ & 31 & 482 & 470 & & & & & & & & \\
\hline 49 & A & 2 & 490 & 478 & & & & & & & & $\omega(\mathrm{CCFeN})_{1}, \omega(\mathrm{CCCC})_{1}-$ out of $\mathrm{pl}$ \\
\hline 50 & A & 2500 & 507 & 494 & & & & & & \multirow{2}{*}{501} & \multirow{2}{*}{508} & \multirow{2}{*}{$v_{\text {as }}(\mathrm{FeN})_{c}, \delta(\mathrm{CCC})_{c}, \delta(\mathrm{CNC})_{c}-$ in pl. } \\
\hline 51 & B & 14 & 518 & 505 & & & & & & & & \\
\hline 52 & $\mathrm{~B}$ & 8 & 523 & 510 & & & & & & & & \multirow{2}{*}{$\tau(\mathrm{CCNFe})_{1}, \omega(\mathrm{CCFeN})_{1}-$ out of $\mathrm{pl}$} \\
\hline 53 & $A$ & 8 & 523 & 510 & & & & & & & & \\
\hline
\end{tabular}




\begin{tabular}{|c|c|c|c|c|c|c|c|c|c|c|c|c|}
\hline \multicolumn{5}{|c|}{$4 a$} & \multicolumn{5}{|c|}{$4 b$} & \multirow{3}{*}{$\begin{array}{c}\text { Calc. } \\
\text { spectral } \\
\text { band }\end{array}$} & \multirow{3}{*}{$\begin{array}{c}\text { Exp.785 } \\
\text { nm } \\
\text { excit. }\end{array}$} & \multirow{3}{*}{ Vibrational mode assignment } \\
\hline \multirow[t]{2}{*}{ No. } & \multirow[t]{2}{*}{ Sym. } & \multirow{2}{*}{$\begin{array}{c}\text { Calc. } \\
\text { Raman } \\
\text { inten. }\end{array}$} & \multicolumn{2}{|c|}{ Calc. wavenumbers } & \multirow[t]{2}{*}{ No. } & \multirow[t]{2}{*}{ Sym. } & \multirow{2}{*}{$\begin{array}{c}\text { Calc. } \\
\text { Raman } \\
\text { inten. }\end{array}$} & \multicolumn{2}{|c|}{ Calc. wavenumbers } & & & \\
\hline & & & $\begin{array}{l}\text { non } \\
\text { scaled }\end{array}$ & $\begin{array}{c}\text { scaled } \\
f=0,975\end{array}$ & & & & $\begin{array}{c}\text { non } \\
\text { scaled }\end{array}$ & $\begin{array}{c}\text { scaled } \\
f=0,972\end{array}$ & & & \\
\hline 54 & B & 28 & 573 & 559 & & & & & & \multirow{4}{*}{573} & \multirow{4}{*}{584} & \multirow{2}{*}{$\tau(\mathrm{SCCC})_{\mathrm{T}}, \omega\left(\mathrm{C}_{\beta} \mathrm{C}_{\beta} \mathrm{C}_{\alpha} \mathrm{C}_{\mathrm{r}}\right)_{\mathrm{T}-\mathrm{c}}-$ out of pl. } \\
\hline 55 & $A$ & 36 & 573 & 559 & & & & & & & & \\
\hline 56 & $A$ & 746 & 585 & 570 & & & & & & & & \multirow{2}{*}{$\mathrm{v}_{\mathrm{S}}(\mathrm{SC})_{\mathrm{T}}, \delta(\mathrm{CSC})_{T}, \delta\left(\mathrm{C}_{\alpha} \mathrm{C}_{\alpha} \mathrm{C}_{\beta}\right)_{\mathrm{C}-\mathrm{T}}, \delta\left(N \mathrm{NC}_{\alpha} \mathrm{C}_{\alpha}\right)_{\mathrm{C}-\mathrm{I}}-$ in pl. } \\
\hline 57 & B & 62 & 585 & 570 & & & & & & & & \\
\hline 58 & A & 2502 & 635 & 619 & & & & & & \multirow{2}{*}{622} & \multirow{2}{*}{636} & \multirow{2}{*}{$v_{S}(S C)_{T}, \delta(C S C)_{T}, v_{S}(F e N)_{c}-$ in pl. } \\
\hline 59 & B & 97 & 637 & 621 & & & & & & & & \\
\hline 60 & $\mathrm{~B}$ & 1 & 647 & 631 & & & & & & & & \multirow{2}{*}{$\omega\left(C_{\beta} C_{\alpha} N C_{\alpha}\right)_{c-1}-$ out of pl. } \\
\hline 61 & A & 4 & 649 & 633 & & & & & & & & \\
\hline 62 & B & 656 & 660 & 644 & & & & & & \multirow{8}{*}{646} & \multirow{8}{*}{654} & $\delta(\mathrm{CNC})_{1}, \delta(\mathrm{CCC})_{1}-$ in pl. \\
\hline 63 & A & 207 & 662 & 645 & & & & & & & & $\begin{array}{l}\delta(\mathrm{CNC})_{1}, \delta(\mathrm{CCC})_{1}, \omega\left(\mathrm{C}_{\beta} \mathrm{C}_{\gamma} \mathrm{C}_{\alpha} \mathrm{C}_{\beta}\right)_{\mathrm{C}-\mathrm{T}}-\text { in }+ \text { (out of) } \\
\text { pl. }\end{array}$ \\
\hline 64 & $\mathrm{~B}$ & 37 & 663 & 646 & & & & & & & & \multirow{2}{*}{$\delta(C N C)_{1}, \delta(C C C)_{1}, \omega\left(C_{\beta} C_{r} C_{\alpha} C_{\beta}\right)_{C-T}-$ in + out of pl. } \\
\hline 65 & A & 409 & 663 & 646 & & & & & & & & \\
\hline 66 & B & 54 & 665 & 648 & & & & & & & & \multirow{2}{*}{$\tau(\mathrm{HCCN})_{C}, \tau(\mathrm{HCSC})_{T}, \omega\left(\mathrm{C}_{\beta} \mathrm{C}_{\gamma} \mathrm{C}_{\alpha} \mathrm{C}_{\beta}\right)_{\mathrm{c}-\mathrm{T}}-$ out of pl. } \\
\hline 67 & A & 73 & 665 & 648 & & & & & & & & \\
\hline 68 & A & 27 & 672 & 655 & & & & & & & & \multirow{2}{*}{$\delta(\mathrm{NCC})_{c}, \delta(\mathrm{CCC})_{c}, \delta(\mathrm{NCC})_{1}-$ in pl. } \\
\hline 69 & B & 21 & 672 & 655 & & & & & & & & \\
\hline 70 & A & 575 & 700 & 683 & & & & & & \multirow{4}{*}{690} & \multirow{4}{*}{692} & $v_{s}(\mathrm{FeN})_{c}, v(\mathrm{SC})_{T}, \delta(\mathrm{CCC})_{c}, \delta(\mathrm{CNC})_{c}, \delta(\mathrm{NCC})_{1}-$ in pl. \\
\hline 71 & B & 43 & 704 & 686 & & & & & & & & $\begin{array}{l}v_{\text {as }}(\mathrm{FeN})_{\mathrm{C}}, \mathrm{V}_{\mathrm{as}}(\mathrm{SC})_{T}, \delta(\mathrm{CCC})_{\mathrm{c}, 1}, \delta(\mathrm{CNC})_{c}, \delta(\mathrm{NCC})_{1}-\text { in } \\
\text { pl. }\end{array}$ \\
\hline 72 & A & 740 & 710 & 692 & & & & & & & & 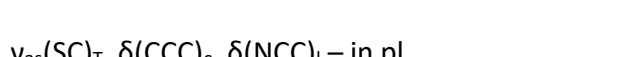 \\
\hline 73 & $B$ & 162 & 711 & 693 & & & & & & & & 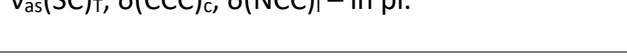 \\
\hline 74 & $B$ & 51 & 759 & 740 & & & & & & & & $\mathrm{~T}(\mathrm{HCSC})_{-}-$unt of $\mathrm{nl}$ \\
\hline 75 & A & 52 & 759 & 740 & & & & & & & & 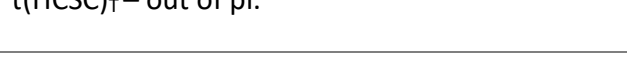 \\
\hline 76 & B & 12 & 767 & 748 & & & & & & 748 & & \\
\hline 77 & A & 12 & 767 & 748 & & & & & & 148 & 154 & 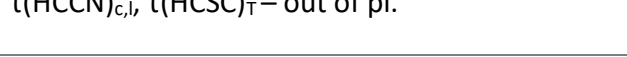 \\
\hline 78 & A & 61 & 775 & 756 & & & & & & & & $2 n !$ \\
\hline 79 & $B$ & 2 & 776 & 757 & & & & & & & & pi. \\
\hline
\end{tabular}




\begin{tabular}{|c|c|c|c|c|c|c|c|c|c|c|c|c|}
\hline \multicolumn{5}{|c|}{$4 a$} & \multicolumn{5}{|c|}{$4 b$} & \multirow{3}{*}{$\begin{array}{c}\text { Calc. } \\
\text { spectral } \\
\text { band }\end{array}$} & \multirow{3}{*}{$\begin{array}{c}\text { Exp.785 } \\
\text { nm } \\
\text { excit. }\end{array}$} & \multirow{3}{*}{ Vibrational mode assignment } \\
\hline \multirow[t]{2}{*}{ No. } & \multirow[t]{2}{*}{ Sym. } & \multirow{2}{*}{$\begin{array}{c}\text { Calc. } \\
\text { Raman } \\
\text { inten. }\end{array}$} & \multicolumn{2}{|c|}{ Calc. wavenumbers } & \multirow[t]{2}{*}{ No. } & \multirow[t]{2}{*}{ Sym. } & \multirow{2}{*}{$\begin{array}{l}\text { Calc. } \\
\text { Raman } \\
\text { inten. }\end{array}$} & \multicolumn{2}{|c|}{ Calc. wavenumbers } & & & \\
\hline & & & $\begin{array}{c}\text { non } \\
\text { scaled }\end{array}$ & $\begin{array}{c}\text { scaled } \\
f=0,975\end{array}$ & & & & $\begin{array}{l}\text { non } \\
\text { scaled }\end{array}$ & $\begin{array}{c}\text { scaled } \\
f=0,972\end{array}$ & & & \\
\hline 80 & B & 0 & 783 & 763 & & & & & & & & \multirow{8}{*}{$\tau(\mathrm{HCCN})_{c, 1}, \tau(\mathrm{HCCC})_{c, 1}-$ out of pl. } \\
\hline 81 & A & 1 & 793 & 773 & & & & & & & & \\
\hline 82 & $B$ & 1 & 794 & 774 & & & & & & & & \\
\hline 83 & A & 1 & 794 & 774 & & & & & & & & \\
\hline 84 & B & 0 & 825 & 804 & & & & & & & & \\
\hline 85 & A & 24 & 828 & 807 & & & & & & & & \\
\hline 86 & $A$ & 2 & 831 & 810 & & & & & & & & \\
\hline 87 & B & 2 & 832 & 811 & & & & & & & & \\
\hline 88 & $\mathrm{~B}$ & 128 & 848 & 827 & & & & & & \multirow{2}{*}{828} & \multirow{4}{*}{849} & \multirow{2}{*}{$v(S C)_{T}, \delta(C C S)_{T}-$ in pl. } \\
\hline 89 & A & 148 & 849 & 828 & & & & & & & & \\
\hline 90 & B & 96 & 865 & 843 & & & & & & \multirow{2}{*}{846} & & \multirow{2}{*}{$\begin{array}{l}v(\mathrm{CC})_{\mathrm{c}-1,} \delta(\mathrm{NCC})_{c}, \delta(\mathrm{CCC})_{c}, \delta(\mathrm{NCC})_{c}, \delta(\mathrm{CCS})_{T}-\text { in } \\
\text { pl. }\end{array}$} \\
\hline 91 & A & 170 & 865 & 843 & & & & & & & & \\
\hline 92 & B & 0 & 888 & 866 & & & & & & & & \multirow{2}{*}{$\tau(\mathrm{HCCC})_{T}, \tau(\mathrm{CCCC})_{T}-$ out of pl. } \\
\hline 93 & A & 7 & 888 & 866 & & & & & & & & \\
\hline 94 & $\mathrm{~B}$ & 0 & 936 & 913 & & & & & & & & \multirow{8}{*}{$\tau(\mathrm{HCCC})_{c, l}, \tau(\mathrm{HCCN})_{c, l},-$ out of pl. } \\
\hline 95 & $A$ & 3 & 937 & 914 & & & & & & & & \\
\hline 96 & $\mathrm{~B}$ & 0 & 937 & 914 & & & & & & & & \\
\hline 97 & A & 0 & 938 & 915 & & & & & & & & \\
\hline 98 & $\mathrm{~B}$ & 1 & 940 & 917 & & & & & & & & \\
\hline 99 & A & 1 & 940 & 917 & & & & & & & & \\
\hline 100 & A & 18 & 941 & 917 & & & & & & & & \\
\hline 101 & $\mathrm{~B}$ & 0 & 943 & 919 & & & & & & & & \\
\hline 102 & A & 34 & 976 & 952 & & & & & & \multirow{4}{*}{977} & & \multirow{2}{*}{$\tau(\mathrm{HCCS})_{T}, \tau(\mathrm{CCCS})_{T}-$ out of pl. } \\
\hline 103 & $\mathrm{~B}$ & 34 & 976 & 952 & & & & & & & 996 & \\
\hline 104 & $\mathrm{~B}$ & 557 & 998 & 973 & & & & & & & 570 & \multirow{2}{*}{$\begin{array}{l}v(\mathrm{CC})_{c, l}, v(\mathrm{NC})_{c, l}, \delta(\mathrm{CCS})_{T}-\text { breathing vibration } \\
\text { tpy - in pl. }\end{array}$} \\
\hline 105 & $A$ & 1194 & 1000 & 975 & & & & & & & & \\
\hline
\end{tabular}




\begin{tabular}{|c|c|c|c|c|c|c|c|c|c|c|c|c|}
\hline \multicolumn{5}{|c|}{$4 a$} & \multicolumn{5}{|c|}{$4 b$} & \multirow{3}{*}{$\begin{array}{l}\text { Calc. } \\
\text { spectral } \\
\text { band }\end{array}$} & \multirow{3}{*}{$\begin{array}{l}\text { Exp.785 } \\
\text { nm } \\
\text { excit. }\end{array}$} & \multirow{3}{*}{ Vibrational mode assignment } \\
\hline \multirow[t]{2}{*}{ No. } & \multirow[t]{2}{*}{ Sym. } & \multirow{2}{*}{$\begin{array}{l}\text { Calc. } \\
\text { Raman } \\
\text { inten. }\end{array}$} & \multicolumn{2}{|c|}{ Calc. wavenumbers } & \multirow[t]{2}{*}{ No. } & \multirow[t]{2}{*}{ Sym. } & \multirow{2}{*}{$\begin{array}{l}\text { Calc. } \\
\text { Raman } \\
\text { inten. }\end{array}$} & \multicolumn{2}{|c|}{ Calc. wavenumbers } & & & \\
\hline & & & $\begin{array}{c}\text { non } \\
\text { scaled }\end{array}$ & $\begin{array}{c}\text { scaled } \\
f=0,975\end{array}$ & & & & $\begin{array}{l}\text { non } \\
\text { scaled }\end{array}$ & $\begin{array}{c}\text { scaled } \\
f=0,972\end{array}$ & & & \\
\hline 106 & A & 4 & 1014 & 989 & & & & & & & & \multirow{4}{*}{$\tau(\mathrm{HCCC})_{1}-$ out of $\mathrm{pl}$} \\
\hline 107 & $\mathrm{~B}$ & 2 & 1014 & 989 & & & & & & & & \\
\hline 108 & B & 2 & 1015 & 990 & & & & & & & & \\
\hline 109 & A & 4 & 1015 & 990 & & & & & & & & \\
\hline 110 & $\mathrm{~B}$ & 42 & 1030 & 1004 & & & & & & \multirow{4}{*}{1011} & \multirow{4}{*}{1021} & \multirow{2}{*}{$\begin{array}{l}v_{\text {as }}(\mathrm{FeN})_{1}, \delta(\mathrm{CCC})_{1}, \delta(\mathrm{CNC})_{1} \text { triangular } \\
\text { breathing vibration I units -out-of-phase - in } \\
\text { pl. }\end{array}$} \\
\hline 111 & A & 18 & 1030 & 1004 & & & & & & & & \\
\hline 112 & $\mathrm{~B}$ & 1553 & 1033 & 1007 & & & & & & & & $v(\mathrm{FeN})_{c, l}, \delta(\mathrm{CCC})_{1, c}, \delta(\mathrm{CNC})_{1, c}, v\left(\mathrm{C}_{\beta} \mathrm{C}_{Y}\right)_{\mathrm{c}} \delta(\mathrm{SCC})_{T}$ \\
\hline 113 & A & 5539 & 1038 & 1012 & & & & & & & & $\begin{array}{l}\text { breathing vibration of c units -in phase- in } \\
\text { pl. }\end{array}$ \\
\hline 114 & A & 18891 & 1056 & 1030 & & & & & & \multirow{5}{*}{1030} & \multirow{5}{*}{1043} & $\begin{array}{l}v_{s}(\mathrm{FeN}) \mathrm{c}, \delta(\mathrm{CNC})_{c}, \delta(\mathrm{NCC})_{c}, \delta(\mathrm{CCC})_{c}- \\
\text { triangular breathing vibration } \mathrm{c} \text { units - in pl. }\end{array}$ \\
\hline 115 & B & 2 & 1056 & 1030 & & & & & & & & \\
\hline 116 & A & 424 & 1056 & 1030 & & & & & & & & \\
\hline 117 & $\mathrm{~B}$ & 2 & 1056 & 1030 & & & & & & & & $\tau(\mathrm{HCCC})_{1}-$ out of pl. \\
\hline 118 & A & 80 & 1056 & 1030 & & & & & & & & \\
\hline 119 & B & 128 & 1057 & 1031 & & & & & & & & $\begin{array}{l}\mathrm{V}_{\mathrm{as}}(\mathrm{FeN})_{c}, \delta(\mathrm{CCC})_{c}, \delta(\mathrm{CNC})_{c}, \delta\left(\mathrm{HC}_{\beta} \mathrm{C}_{\beta}\right)_{T}- \\
\text { triangular breathing vibration c units - in pl. }\end{array}$ \\
\hline 120 & $\mathrm{~B}$ & 44 & 1072 & 1045 & & & & & & & & \\
\hline 121 & A & 16 & 1074 & 1047 & & & & & & & & $v(C C)_{1} \delta\left(\mathrm{HCC}_{1}-\right.$ in-nl \\
\hline 122 & B & 1 & 1075 & 1048 & & & & & & & & 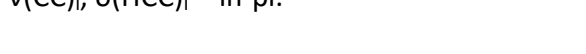 \\
\hline 123 & A & 98 & 1075 & 1048 & & & & & & $10595 n$ & $1059 s n$ & \\
\hline 124 & A & 106 & 1092 & 1065 & & & & & & & & $v\left(C_{\beta} C_{\beta}\right)_{T}, \delta(H C S)_{T}, \delta(N C C)_{c, l}, \delta(H C C)_{C, 1}-$ \\
\hline 125 & $\mathrm{~B}$ & 27 & 1092 & 1065 & & & & & & & & breathing vibration of T - in-pl. \\
\hline 126 & B & 39 & 1110 & 1082 & & & & & & 1084 & 1077 & $v(\mathrm{NC}) \delta(\mathrm{HCCl}) \delta(\mathrm{HCS})_{-} \delta(\mathrm{HCOC})_{-}-$in $-n l$ \\
\hline 127 & A & 583 & 1112 & 1084 & & & & & & 1004 & 年 & 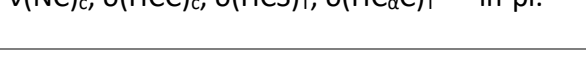 \\
\hline 128 & B & 7 & 1128 & 1100 & & & & & & & & \\
\hline 129 & $A$ & 49 & 1128 & 1100 & & & & & & & & $\delta\left(\mathrm{HC}_{\beta} \mathrm{C}\right)_{c, 1}-$ in-pl. \\
\hline
\end{tabular}




\begin{tabular}{|c|c|c|c|c|c|c|c|c|c|c|c|c|}
\hline \multicolumn{5}{|c|}{$4 a$} & \multicolumn{5}{|c|}{$4 b$} & \multirow{3}{*}{$\begin{array}{c}\text { Calc. } \\
\text { spectral } \\
\text { band }\end{array}$} & \multirow{3}{*}{$\begin{array}{c}\text { Exp.785 } \\
\text { nm } \\
\text { excit. }\end{array}$} & \multirow{3}{*}{ Vibrational mode assignment } \\
\hline \multirow[t]{2}{*}{ No. } & \multirow[t]{2}{*}{ Sym. } & \multirow{2}{*}{$\begin{array}{c}\text { Calc. } \\
\text { Raman } \\
\text { inten. }\end{array}$} & \multicolumn{2}{|c|}{ Calc. wavenumbers } & \multirow[t]{2}{*}{ No. } & \multirow[t]{2}{*}{ Sym. } & \multirow{2}{*}{$\begin{array}{c}\text { Calc. } \\
\text { Raman } \\
\text { inten. }\end{array}$} & \multicolumn{2}{|c|}{ Calc. wavenumbers } & & & \\
\hline & & & $\begin{array}{l}\text { non } \\
\text { scaled }\end{array}$ & $\begin{array}{c}\text { scaled } \\
f=0,975\end{array}$ & & & & $\begin{array}{l}\text { non } \\
\text { scaled }\end{array}$ & $\begin{array}{c}\text { scaled } \\
f=0,972\end{array}$ & & & \\
\hline 130 & B & 48 & 1136 & 1108 & & & & & & \multirow{4}{*}{1113} & \multirow{4}{*}{1118} & \multirow{2}{*}{$\delta\left(\mathrm{HC}_{\alpha} \mathrm{S}\right)_{T}, \delta\left(\mathrm{HC}_{\beta} \mathrm{C}\right)_{T}-$ in-pl. } \\
\hline 131 & $A$ & 767 & 1137 & 1109 & & & & & & & & \\
\hline 132 & $A$ & 1475 & 1142 & 1113 & & & & & & & & \multirow{2}{*}{$\mathrm{v}\left(\mathrm{C}_{\alpha} \mathrm{C}_{\beta}\right)_{1} \delta(\mathrm{HCC})_{1}, \delta(\mathrm{HCC})_{T}-$ in-pl. } \\
\hline 133 & B & 77 & 1145 & 1116 & & & & & & & & \\
\hline 134 & B & 14 & 1195 & 1165 & & & & & & & & \multirow{2}{*}{$v(C N)_{c, l}, \delta(H C C)_{c, l}, \delta(H C C)_{T}-$ in-pl. } \\
\hline 135 & A & 19 & 1195 & 1165 & & & & & & & & \\
\hline 136 & B & 8 & 1214 & 1184 & & & & & & \multirow{4}{*}{1184} & \multirow{4}{*}{1166} & \multirow{4}{*}{$\delta(\mathrm{HCC})_{1}-$ in-pl. } \\
\hline 137 & $A$ & 66 & 1214 & 1184 & & & & & & & & \\
\hline 138 & B & 4 & 1215 & 1185 & & & & & & & & \\
\hline 139 & A & 204 & 1215 & 1185 & & & & & & & & \\
\hline 140 & $\mathrm{~B}$ & 163 & 1244 & 1213 & & & & & & \multirow{2}{*}{1215} & \multirow{2}{*}{1214} & \multirow{2}{*}{$\begin{array}{l}v(C C)_{C-T}, \delta\left(C_{Y-C} C_{\alpha} C_{\beta}\right)_{T-C,} \delta\left(H C_{\beta} C_{\alpha}\right)_{T}, \delta(H C C)_{c}- \\
\text { in-pl. }\end{array}$} \\
\hline 141 & $A$ & 464 & 1244 & 1213 & & & & & & & & \\
\hline 142 & B & 428 & 1291 & 1259 & & & & & & \multirow[b]{2}{*}{1259} & \multirow{2}{*}{1243} & \multirow[b]{2}{*}{$\delta(\mathrm{HCC})_{T}, \mathrm{v}(\mathrm{CC})_{\mathrm{c}-\mathrm{T}}, \delta(\mathrm{HCC})_{\mathrm{c}}-$ in-pl. } \\
\hline 143 & A & 620 & 1292 & 1260 & & & & & & & & \\
\hline 144 & $\mathrm{~B}$ & 349 & 1312 & 1279 & & & & & & & & \\
\hline 145 & A & 377 & 1312 & 1279 & & & & & & $3 \mathrm{ch}$ & $1282 \mathrm{ch}$ & $V_{\text {as }}(N C)_{1}, v\left(C_{\beta} C_{\gamma}\right)_{1} \delta(H C C)_{c},-$ In-pl. \\
\hline 146 & B & 128 & 1314 & 1281 & & & & & & ( & 1202511 & \\
\hline 147 & A & 132 & 1314 & 1281 & & & & & & & & $v_{a s}(N C)_{c}, v(C C)_{c-1}, \delta(H C N)_{l}, \delta(H C C)_{c}-$ in-pl. \\
\hline 148 & A & 112 & 1317 & 1284 & & & & & & & & $\begin{array}{l}v_{\text {as }}(\mathrm{NC})_{1}, v\left(C_{\alpha} C_{\beta}\right)_{1}, \delta(H C C)_{c}, \delta(H C S)_{T}, \delta(H C C)_{T}- \\
\text { in-pl. }\end{array}$ \\
\hline 149 & B & 55 & 1319 & 1286 & & & & & & 98 & 1293 & $v_{\text {as }}(N C)_{1}, v\left(C_{\alpha} C_{\beta}\right)_{1}, \delta(H C C)_{c}-$ in-pl. \\
\hline 150 & $\mathrm{~B}$ & 31 & 1326 & 1293 & & & & & & & & 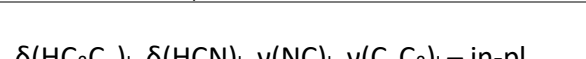 \\
\hline 151 & A & 3320 & 1329 & 1296 & & & & & & & & 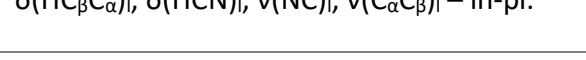 \\
\hline 152 & A & 725 & 1339 & 1306 & & & & & & & & \\
\hline 153 & $\mathrm{~B}$ & 129 & 1339 & 1306 & & & & & & 1307 & $1300 \mathrm{sh}$ & 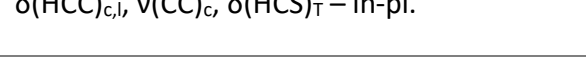 \\
\hline 154 & A & 116 & 1343 & 1309 & & & & & & 100 & $1000 \mathrm{sin}$ & \\
\hline 155 & $\mathrm{~B}$ & 9 & 1343 & 1309 & & & & & & & & $O(H C C), v(C I N)|, V(C C)|-\mid n-p I$. \\
\hline 156 & A & 671 & 1376 & 1342 & & & & & & & & $v(\mathrm{CC})_{T}, \delta(\mathrm{HCS})_{T}, \delta(\mathrm{HCC})_{T}, v_{a s}(\mathrm{NC})_{C}, v(C C)_{C-1}$ \\
\hline 157 & B & 260 & 1376 & 1342 & & & & & & 1342 & 1339 & $\delta(\mathrm{HCC})_{c}-$ in-pl. \\
\hline
\end{tabular}




\begin{tabular}{|c|c|c|c|c|c|c|c|c|c|c|c|c|}
\hline \multicolumn{5}{|c|}{$4 a$} & \multicolumn{5}{|c|}{$4 b$} & \multirow{3}{*}{$\begin{array}{c}\text { Calc. } \\
\text { spectral } \\
\text { band }\end{array}$} & \multirow{3}{*}{$\begin{array}{c}\text { Exp.785 } \\
\text { nm } \\
\text { excit. }\end{array}$} & \multirow{3}{*}{ Vibrational mode assignment } \\
\hline \multirow[t]{2}{*}{ No. } & \multirow[t]{2}{*}{ Sym. } & \multirow{2}{*}{$\begin{array}{c}\text { Calc. } \\
\text { Raman } \\
\text { inten. }\end{array}$} & \multicolumn{2}{|c|}{ Calc. wavenumbers } & \multirow[t]{2}{*}{ No. } & \multirow[t]{2}{*}{ Sym. } & \multirow{2}{*}{$\begin{array}{c}\text { Calc. } \\
\text { Raman } \\
\text { inten. }\end{array}$} & \multicolumn{2}{|c|}{ Calc. wavenumbers } & & & \\
\hline & & & $\begin{array}{l}\text { non } \\
\text { scaled }\end{array}$ & $\begin{array}{c}\text { scaled } \\
f=0,975\end{array}$ & & & & \multicolumn{2}{|c|}{$\begin{array}{cc}\text { non } & \text { scaled } \\
\text { scaled } & f=0,972\end{array}$} & & & \\
\hline $\begin{array}{l}158 \\
159\end{array}$ & $\begin{array}{l}\mathrm{A} \\
\mathrm{B}\end{array}$ & $\begin{array}{c}16960 \\
849\end{array}$ & $\begin{array}{l}1408 \\
1409\end{array}$ & $\begin{array}{l}1373 \\
1374\end{array}$ & 78 & $A^{\prime}$ & 6472 & 1411 & 1376 & 1375 & 1370 & $v_{s}(N C)_{c}, v(C C)_{c-1,} v(C C)_{c-T}, \delta(H C C)_{c, l, T}-$ in-pl. \\
\hline 160 & A & 11549 & 1440 & 1404 & \multirow{2}{*}{79} & \multirow{2}{*}{$A^{\prime}$} & & & & \multirow[b]{2}{*}{1409} & \multirow{2}{*}{1404} & \multirow{2}{*}{$v\left(C_{\beta} C_{\beta}\right)_{T}, \delta(H C C)_{T, c, l}, v_{s}\left(C_{\alpha} C_{\beta}\right)_{c}-$ in-pl. } \\
\hline 161 & $\mathrm{~B}$ & 167 & 1443 & 1407 & & & 4611 & 1444 & 1408 & & & \\
\hline 162 & $\mathrm{~B}$ & 151 & 1467 & 1430 & 80 & $A^{\prime}$ & 388 & 1471 & 1434 & & & $\delta(\mathrm{HCC})_{1}, v_{a s}\left(C_{\alpha} C_{\beta}\right)_{-}-$in-pl. \\
\hline 163 & $A$ & 1019 & 1467 & 1430 & 80 & $n$ & 300 & 14 & 1404 & 1434 & & 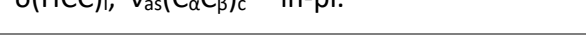 \\
\hline 164 & $\mathrm{~B}$ & 16 & 1475 & 1438 & 81 & $A^{\prime}$ & 5816 & 1474 & 1437 & 1434 & 1438 & $v\left(C_{\alpha} C_{\beta}\right)_{T}, \delta(H C C)_{T}, \delta(H C C)_{1}-$ in-pl. \\
\hline 165 & $\mathrm{~A}$ & 1681 & 1475 & 1438 & & & & & & & & \\
\hline 166 & $\mathrm{~B}$ & 78 & 1480 & 1443 & 82 & $A^{\prime}$ & 455 & 1486 & 1449 & & & $\mathrm{v}_{\mathrm{as}}\left(\mathrm{C}_{\alpha} \mathrm{C}_{\beta}\right)_{\mathrm{c}} \delta(\mathrm{HCC})_{\mathrm{lc}}-$ in pl. \\
\hline 167 & A & 31822 & 1483 & 1446 & 82 & $H$ & 400 & 1400 & שדירו & $1452 \mathrm{sh}$ & $1450 \mathrm{sh}$ & 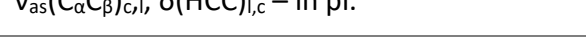 \\
\hline 168 & $\mathrm{~B}$ & 157 & 1493 & 1456 & 83 & $A^{\prime}$ & 660 & 1493 & 1456 & $145 \angle \mathrm{sn}$ & $1450 \mathrm{sn}$ & $v\left(C_{\beta} C_{\beta}\right)_{T}, v(C C)_{c-T}, v_{s}\left(C_{r} C_{\beta}\right)_{c}, \delta(H C C)_{c, l, T}-$ in-pl. \\
\hline 169 & A & 5600 & 1497 & 1460 & & & & & & & & \\
\hline 170 & $\mathrm{~B}$ & 554 & 1510 & 1472 & 84 & $A^{\prime}$ & 2173 & 1518 & 1480 & 1477 & 1473 & $v(C C)_{C-1}, v_{a s}\left(C_{\alpha} C_{\beta}\right)_{1}, \delta(H C C)_{1}-$ in pl. \\
\hline 171 & A & 10604 & 1511 & 1473 & & $A$ & & & 1400 & & & 传 \\
\hline 172 & B & 80 & 1524 & 1486 & 85 & $A^{\prime}$ & 758 & 1528 & 1490 & $1495 \mathrm{sh}$ & $1485 \mathrm{sh}$ & \\
\hline 173 & A & 86 & 1524 & 1486 & 85 & $\mathrm{H}$ & 150 & $10<0$ & 1450 & $14935 \pi$ & $14035 \pi$ & 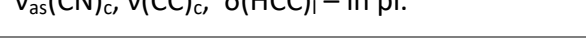 \\
\hline 174 & A & 832 & 1570 & 1531 & 86 & $A^{\prime}$ & 3746 & 1570 & 1531 & 1531 & 1531 & $v(\mathrm{CN})_{c} v(\mathrm{CC})_{c,} v(\mathrm{CC})_{c-1} \delta(\mathrm{HCC})_{c}-$ in pl. \\
\hline 175 & B & 473 & 1570 & 1531 & 86 & & & & & & & (16) \\
\hline 176 & B & 10 & 1583 & 1543 & 87 & $A^{\prime}$ & 854 & 1574 & 1535 & 1525 & 1541 & $v(C \mathrm{CO})_{T} v(\mathrm{C} C \mathrm{C}) \mathrm{v}(\mathrm{CC})-\delta(\mathrm{HCC})_{-}-$in $n$ \\
\hline 177 & A & 10145 & 1584 & 1544 & $8 /$ & A & 854 & $15 / 4$ & 1535 & 1535 & 1541 & $V\left(C_{\alpha} C_{\beta}\right)_{T}, \quad V\left(C_{\alpha} C_{\beta}\right)_{c}, V(C C)_{c-T}, o(H C C)_{c, T}-$ In pl. \\
\hline 178 & $\mathrm{~B}$ & 195 & 1604 & 1564 & 88 & $A^{\prime}$ & 633 & & 15 & & & $v_{\text {as }}\left(C_{r} C_{\beta}\right)_{1}, v_{a s}(N C)_{1}, \delta(H C C)_{1}, \delta(N F e N)_{\text {out-of-ph. }}-$ \\
\hline 179 & A & 196 & 1604 & 1564 & 88 & $A^{\prime}$ & 633 & 160 & 15 & 1560 & 1565 & in pl. \\
\hline 180 & A & 1107 & 1605 & 1565 & 89 & $A^{\prime}$ & 685 & 1603 & 1563 & & & $v_{\text {as }}\left(C_{\gamma} C_{\beta}\right)_{1}, v_{\text {as }}(N C)_{1}, \delta(H C C)_{1}, \delta(N F e N)_{\text {in ph. }}-$ in \\
\hline 181 & $\mathrm{~B}$ & 185 & 1605 & 1565 & 89 & A & 685 & 1603 & 1563 & & & \\
\hline 182 & $\mathrm{~B}$ & 173 & 1644 & 1603 & & & 1754 & 1645 & 1604 & & & $v\left(C_{\alpha} C_{\beta}\right)_{1}, \delta\left(C_{\beta} C_{\gamma} C_{\beta}\right)_{1}, \delta(C N C)_{1}, \delta(H C C)_{1}-I$ units \\
\hline 183 & A & 1454 & 1645 & 1604 & 90 & $A^{\prime}$ & $1 / 54$ & 1645 & 1604 & 1605 & 1605 & out-of-ph - in pl. \\
\hline 184 & $\mathrm{~B}$ & 1281 & 1645 & 1604 & 91 & $A^{\prime}$ & 2 & 1647 & 160 & 1605 & 1605 & $v\left(C_{\alpha} C_{\beta}\right)_{1}, \delta\left(C_{\beta} C_{\gamma} C_{\beta}\right)_{1}, \delta(C N C), \delta(H C C)_{1}-I$ units \\
\hline 185 & A & 7063 & 1647 & 1606 & y1 & A & 2534 & 1047 & 1000 & & & in-ph - in pl. \\
\hline 186 & $\mathrm{~B}$ & 1172 & 1651 & 1610 & 92 & $A^{\prime}$ & 2895 & 1652 & 1611 & 1611 & 1614 & \\
\hline 187 & A & 9137 & 1651 & 1610 & 92 & $A$ & 2095 & 1052 & 1011 & & 1014 & 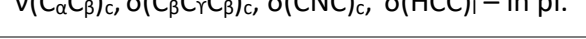 \\
\hline
\end{tabular}

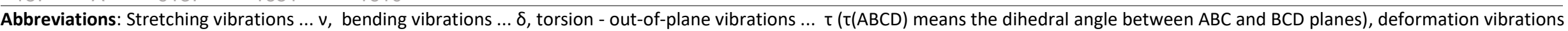

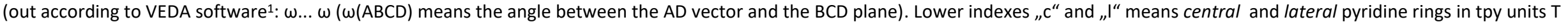

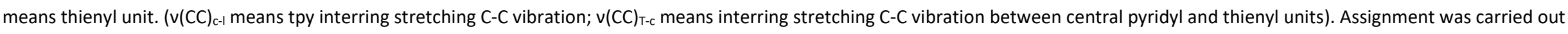

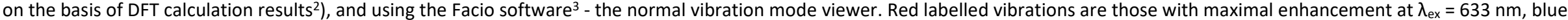
labelled with $\lambda_{\mathrm{ex}}=532 \mathrm{~nm}$. 


\section{References:}

(1) Jamroz, M. H. Vibrational Energy Distribution Analysis (VEDA): Scopes and Limitations. Spectrochim. ACTA PART A-MOLECULAR Biomol. Spectrosc. 2013, 114, 220-230. https://doi.org/10.1016/j.saa.2013.05.096.

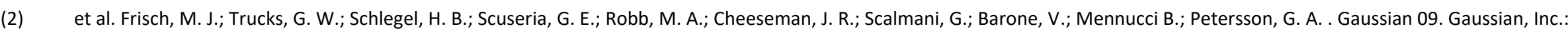
Wallingford CT 2013.

(3) Suenaga, M. Facio. 
Table S3 - List of the first seventeen B3LYP/LANL2-DZ electronic transitions for $\mathbf{2 a}$ calculated by TD-DFT.

\begin{tabular}{|c|c|c|c|c|}
\hline$\#$ & Energy $(\mathrm{eV})$ & Assignment & $f^{a}$ & $\begin{array}{l}\text { Symmetry of } \\
\text { excited state }\end{array}$ \\
\hline 1 & 2.2797 & $\begin{array}{c}\mathrm{H}-14 \rightarrow \mathrm{L}+10(0.11044) \\
\mathrm{H} \rightarrow \mathrm{L}+10(0.67817)\end{array}$ & 0.0000 & $\mathrm{~A} 2$ \\
\hline $2 / 3$ & $\begin{array}{l}2.5838 \\
2.5838\end{array}$ & $\begin{aligned} \mathrm{H}-1 & \rightarrow \mathrm{L}+10(0.36248) \\
\mathrm{H}-1 & \rightarrow \mathrm{L}+11(0.25719) \\
\mathrm{H} & \rightarrow \mathrm{L}(0.52795) \\
\mathrm{H}-2 & \rightarrow \mathrm{L}+10(0.36248) \\
\mathrm{H}-2 & \rightarrow \mathrm{L}+11(-0.25719) \\
\mathrm{H} & \rightarrow \mathrm{L}+1(0.52795)\end{aligned}$ & 0.0057 & $E$ \\
\hline $4 / 5$ & $\begin{array}{l}2.696 \\
2.696\end{array}$ & $\begin{aligned} \mathrm{H}-1 & \rightarrow \mathrm{L}+10(-0.28264) \\
\mathrm{H}-1 & \rightarrow \mathrm{L}+11(-0.43223) \\
& \mathrm{H} \rightarrow \mathrm{L}(0.447) \\
\mathrm{H}-2 & \rightarrow \mathrm{L}+10(-0.28265) \\
\mathrm{H}-2 & \rightarrow \mathrm{L}+11(0.43223) \\
\mathrm{H} & \rightarrow \mathrm{L}+1(0.447)\end{aligned}$ & $\begin{array}{l}0.0014 \\
0.0014\end{array}$ & $E$ \\
\hline 6 & 2.7008 & $\begin{array}{c}\mathrm{H}-2 \rightarrow \mathrm{L}+1(-0.48246) \\
\mathrm{H}-1 \rightarrow \mathrm{L}(0.48246) \\
\mathrm{H} \rightarrow \mathrm{L}+11(0.16164)\end{array}$ & 0.0000 & $E$ \\
\hline 7 & 2.7082 & $\begin{array}{c}\mathrm{H}-2 \rightarrow \mathrm{L}+1(0.4964) \\
\mathrm{H}-1 \rightarrow \mathrm{L}(0.49639)\end{array}$ & 0.0000 & E \\
\hline 8 & 2.8713 & $\begin{array}{c}\mathrm{H}-2 \rightarrow \mathrm{L}(0.4797) \\
\mathrm{H}-1 \rightarrow \mathrm{L}+1(0.4797) \\
\mathrm{H} \rightarrow \mathrm{L}+2(-0.18097)\end{array}$ & 0.0777 & $\mathrm{E}$ \\
\hline 9 & 2.8868 & $\begin{array}{c}\mathrm{H}-2 \rightarrow \mathrm{L}(0.32244) \\
\mathrm{H}-1 \rightarrow \mathrm{L}+1(-0.32244) \\
\mathrm{H} \rightarrow \mathrm{L}+3(0.52949)\end{array}$ & 0.0000 & A1 \\
\hline $10 / 11$ & 2.969 & $\begin{array}{c}\mathrm{H}-2 \rightarrow \mathrm{L}+3(0.66848) \\
\mathrm{H}-2 \rightarrow \mathrm{L}+5(-0.10891) \\
\mathrm{H}-1 \rightarrow \mathrm{L}+10(-0.11327) \\
\mathrm{H}-1 \rightarrow \mathrm{L}+11(0.13443) \\
\mathrm{H}-2 \rightarrow \mathrm{L}+10(0.11327) \\
\mathrm{H}-2 \rightarrow \mathrm{L}+11(0.13443) \\
\mathrm{H}-1 \rightarrow \mathrm{L}+3(0.66848) \\
\mathrm{H}-1 \rightarrow \mathrm{L}+5(-0.10891)\end{array}$ & 0.0107 & $E$ \\
\hline 12 & 3.0564 & $\begin{array}{c}\mathrm{H}-2 \rightarrow \mathrm{L}(0.12533) \\
\mathrm{H}-1 \rightarrow \mathrm{L}+1(0.12533) \\
\mathrm{H} \rightarrow \mathrm{L}+2(0.67898)\end{array}$ & 0.0669 & B2 \\
\hline $13 / 14$ & $\begin{array}{l}3.1002 \\
3.1002 \\
\end{array}$ & $\begin{array}{l}\mathrm{H}-2 \rightarrow \mathrm{L}+2(0.70162) \\
\mathrm{H}-1 \rightarrow \mathrm{L}+2(0.70162)\end{array}$ & $\begin{array}{l}0.0101 \\
0.0101\end{array}$ & $\begin{array}{l}E \\
E\end{array}$ \\
\hline 15 & 3.3962 & $\begin{array}{c}\mathrm{H}-2 \rightarrow \mathrm{L}(-0.33954) \\
\mathrm{H}-1 \rightarrow \mathrm{L}+1(0.33954) \\
\mathrm{H} \rightarrow \mathrm{L}+3(0.45167) \\
\mathrm{H} \rightarrow \mathrm{L}+5(0.20536)\end{array}$ & 0.0000 & A1 \\
\hline
\end{tabular}




\begin{tabular}{|c|c|c|c|c|}
\hline $16 / 17$ & $\begin{array}{l}3.5765 \\
\\
3.5765\end{array}$ & $\begin{aligned} \mathrm{H}-2 & \rightarrow \mathrm{L}+3(0.20377) \\
\mathrm{H}-2 & \rightarrow \mathrm{L}+5(0.15156) \\
\mathrm{H}-1 & \rightarrow \mathrm{L}+10(0.47425) \\
\mathrm{H}-1 & \rightarrow \mathrm{L}+11(-0.4033) \\
\mathrm{H} & \rightarrow \mathrm{L}(-0.12538) \\
\mathrm{H}-2 & \rightarrow \mathrm{L}+10(0.47425) \\
\mathrm{H}-2 & \rightarrow \mathrm{L}+11(0.4033) \\
\mathrm{H}-1 & \rightarrow \mathrm{L}+3(-0.20377) \\
\mathrm{H}-1 & \rightarrow \mathrm{L}+5(-0.15156) \\
\mathrm{H} & \rightarrow \mathrm{L}+1(-0.12538)\end{aligned}$ & 0.0000 & E \\
\hline
\end{tabular}

${ }^{a}$ Oscillator strength. 
Table S4 - List of the first seventeen B3LYP/LANL2-DZ electronic transitions for 4a calculated by TD-DFT.

\begin{tabular}{|c|c|c|c|c|}
\hline \# & Energy (eV) & Assignment & $f^{a}$ & $\begin{array}{l}\text { Symmetry of } \\
\text { excited state }\end{array}$ \\
\hline 1 & 2.289 & $\begin{array}{c}\mathrm{H}-18 \rightarrow \mathrm{L}+10(0.10422) \\
\mathrm{H}-2 \rightarrow \mathrm{L}+10(0.6748) \\
\mathrm{H}-2 \rightarrow \mathrm{L}+16(-0.10336)\end{array}$ & 0.0000 & $\mathrm{~B}$ \\
\hline 2 & 2.5153 & $\begin{array}{c}\mathrm{H}-4 \rightarrow \mathrm{L}+1(0.20823) \\
\mathrm{H}-3 \rightarrow \mathrm{L}(-0.20766) \\
\mathrm{H}-1 \rightarrow \mathrm{L}(-0.44428) \\
\mathrm{H} \rightarrow \mathrm{L}+1(0.44451)\end{array}$ & 0.0000 & $\mathrm{~B}$ \\
\hline 3 & 2.5184 & $\begin{array}{c}\mathrm{H}-4 \rightarrow \mathrm{L}(0.20522) \\
\mathrm{H}-3 \rightarrow \mathrm{L}+1(-0.20457) \\
\mathrm{H}-2 \rightarrow \mathrm{L}+12(0.11875) \\
\mathrm{H}-1 \rightarrow \mathrm{L}+1(-0.43997) \\
\mathrm{H} \rightarrow \mathrm{L}(0.44037)\end{array}$ & 0.0000 & A \\
\hline 4 & 2.5533 & $\begin{array}{c}\mathrm{H}-4 \rightarrow \mathrm{L}+12(0.17494) \\
\mathrm{H}-3 \rightarrow \mathrm{L}+10(0.25699) \\
\mathrm{H}-2 \rightarrow \mathrm{L}(0.52794) \\
\mathrm{H}-1 \rightarrow \mathrm{L}+10(0.2553) \\
\mathrm{H} \rightarrow \mathrm{L}+12(0.16831)\end{array}$ & 0.0065 & A \\
\hline 5 & 2.5534 & $\begin{array}{c}\mathrm{H}-4 \rightarrow \mathrm{L}+10(-0.25801) \\
\mathrm{H}-3 \rightarrow \mathrm{L}+12(-0.17426) \\
\mathrm{H}-2 \rightarrow \mathrm{L}+1(0.52773) \\
\mathrm{H}-1 \rightarrow \mathrm{L}+12(-0.16834) \\
\mathrm{H} \rightarrow \mathrm{L}+10(-0.25536)\end{array}$ & 0.0065 & B \\
\hline 6 & 2.6479 & $\begin{array}{c}\mathrm{H}-4 \rightarrow \mathrm{L}+1(0.17422) \\
\mathrm{H}-3 \rightarrow \mathrm{L}(0.1737) \\
\mathrm{H}-1 \rightarrow \mathrm{L}(0.45778) \\
\mathrm{H} \rightarrow \mathrm{L}+1(0.45772)\end{array}$ & 0.5046 & B \\
\hline 7 & 2.6735 & $\begin{array}{c}\mathrm{H}-6 \rightarrow \mathrm{L}+12(-0.10582) \\
\mathrm{H}-4 \rightarrow \mathrm{L}+12(-0.29905) \\
\mathrm{H}-3 \rightarrow \mathrm{L}+10(-0.20996) \\
\mathrm{H}-2 \rightarrow \mathrm{L}(0.4453) \\
\mathrm{H}-1 \rightarrow \mathrm{L}+10(-0.21094) \\
\mathrm{H} \rightarrow \mathrm{L}+12(-0.29064)\end{array}$ & 0.0012 & A \\
\hline 8 & 2.6736 & $\begin{array}{c}\mathrm{H}-5 \rightarrow \mathrm{L}+12(0.10894) \\
\mathrm{H}-4 \rightarrow \mathrm{L}+10(0.21057) \\
\mathrm{H}-3 \rightarrow \mathrm{L}+12(0.29783) \\
\mathrm{H}-2 \rightarrow \mathrm{L}+1(0.44544) \\
\mathrm{H}-1 \rightarrow \mathrm{L}+12(0.2905) \\
\mathrm{H} \rightarrow \mathrm{L}+10(0.21078)\end{array}$ & 0.0015 & B \\
\hline 9 & 2.7795 & $\begin{array}{c}\mathrm{H}-4 \rightarrow \mathrm{L}+3(0.14542) \\
\mathrm{H}-2 \rightarrow \mathrm{L}+3(0.271) \\
\mathrm{H}-1 \rightarrow \mathrm{L}+1(0.26404) \\
\mathrm{H}-1 \rightarrow \mathrm{L}+2(0.13192) \\
\mathrm{H} \rightarrow \mathrm{L}(0.26617) \\
\mathrm{H} \rightarrow \mathrm{L}+3(0.44302)\end{array}$ & 0.0060 & A \\
\hline
\end{tabular}




\begin{tabular}{|c|c|c|c|c|}
\hline 10 & 2.7832 & $\begin{array}{c}\mathrm{H}-3 \rightarrow \mathrm{L}+3(0.2081) \\
\mathrm{H}-1 \rightarrow \mathrm{L}+3(0.62109) \\
\mathrm{H} \rightarrow \mathrm{L}+2(0.18122)\end{array}$ & 0.0092 & $\mathrm{~B}$ \\
\hline 11 & 2.7867 & $\begin{array}{c}\mathrm{H}-4 \rightarrow \mathrm{L}(-0.10304) \\
\mathrm{H}-4 \rightarrow \mathrm{L}+3(0.15039) \\
\mathrm{H}-3 \rightarrow \mathrm{L}+1(-0.10357) \\
\mathrm{H}-2 \rightarrow \mathrm{L}+3(-0.28782) \\
\mathrm{H}-1 \rightarrow \mathrm{L}+1(-0.2642) \\
\mathrm{H}-1 \rightarrow \mathrm{L}+2(0.12371) \\
\mathrm{H} \rightarrow \mathrm{L}(-0.26163) \\
\mathrm{H} \rightarrow \mathrm{L}+3(0.4354)\end{array}$ & 0.0032 & $\mathrm{~A}$ \\
\hline 12 & 2.8776 & $\begin{array}{c}\mathrm{H}-4 \rightarrow \mathrm{L}+2(0.16555) \\
\mathrm{H}-3 \rightarrow \mathrm{L}+3(-0.11036) \\
\mathrm{H}-1 \rightarrow \mathrm{L}+3(-0.1372) \\
\mathrm{H} \rightarrow \mathrm{L}+2(0.65412)\end{array}$ & 0.0019 & $\mathrm{~B}$ \\
\hline 13 & 2.8777 & $\begin{array}{c}\mathrm{H}-4 \rightarrow \mathrm{L}+3(-0.11043) \\
\mathrm{H}-3 \rightarrow \mathrm{L}+2(0.16505) \\
\mathrm{H}-1 \rightarrow \mathrm{L}+2(0.65412) \\
\mathrm{H} \rightarrow \mathrm{L}+3(-0.13695)\end{array}$ & 0.0019 & A \\
\hline 14 & 3.0709 & $\mathrm{H}-2 \rightarrow \mathrm{L}+2(0.69719)$ & 0.1057 & $\mathrm{~B}$ \\
\hline 15 & 3.2206 & $\begin{array}{c}\mathrm{H}-4 \rightarrow \mathrm{L}(0.19331) \\
\mathrm{H}-3 \rightarrow \mathrm{L}+1(0.19288) \\
\mathrm{H}-2 \rightarrow \mathrm{L}+3(0.46589) \\
\mathrm{H}-1 \rightarrow \mathrm{L}+1(-0.30166) \\
\mathrm{H} \rightarrow \mathrm{L}(-0.30199)\end{array}$ & 0.0001 & A \\
\hline 16 & 3.315 & $\begin{array}{c}\mathrm{H}-6 \rightarrow \mathrm{L}(0.11621) \\
\mathrm{H}-5 \rightarrow \mathrm{L}+1(-0.12064) \\
\mathrm{H}-4 \rightarrow \mathrm{L}(0.41093) \\
\mathrm{H}-3 \rightarrow \mathrm{L}+1(-0.40963) \\
\mathrm{H}-2 \rightarrow \mathrm{L}+12(0.14617) \\
\mathrm{H}-1 \rightarrow \mathrm{L}+1(0.23355) \\
\mathrm{H} \rightarrow \mathrm{L}(-0.23321)\end{array}$ & 0.0000 & $\mathrm{~A}$ \\
\hline 17 & 3.3295 & $\begin{array}{c}\mathrm{H}-6 \rightarrow \mathrm{L}+1(-0.11986) \\
\mathrm{H}-5 \rightarrow \mathrm{L}(0.1242) \\
\mathrm{H}-4 \rightarrow \mathrm{L}+1(-0.42712) \\
\mathrm{H}-3 \rightarrow \mathrm{L}(0.42947) \\
\mathrm{H}-1 \rightarrow \mathrm{L}(-0.22377) \\
\mathrm{H} \rightarrow \mathrm{L}+1(0.2224)\end{array}$ & 0.0000 & $\mathrm{~B}$ \\
\hline
\end{tabular}

${ }^{a}$ Oscillator strength. 